\title{
GLOBAL OPTIMALITY CONDITIONS FOR A DYNAMIC BLOCKING PROBLEM
}

\author{
Alberto Bressan ${ }^{1}$ and TaO WAng ${ }^{1}$
}

\begin{abstract}
The paper is concerned with a class of optimal blocking problems in the plane. We consider a time dependent set $R(t) \subset \mathbb{R}^{2}$, described as the reachable set for a differential inclusion. To restrict its growth, a barrier $\Gamma$ can be constructed, in real time. This is a one-dimensional rectifiable set which blocks the trajectories of the differential inclusion. In this paper we introduce a definition of "regular strategy", based on a careful classification of blocking arcs. Moreover, we derive local and global necessary conditions for an optimal strategy, which minimizes the total value of the burned region plus the cost of constructing the barrier. We show that a Lagrange multiplier, corresponding to the constraint on the construction speed, can be interpreted as the "instantaneous value of time". This value, which we compute by two separate formulas, remains constant when free arcs are constructed and is monotone decreasing otherwise.
\end{abstract}

Mathematics Subject Classification. 49J24, 49K24.

Received March 11, 2010.

Published online December 23, 2010.

\section{INTRODUCTION}

Aim of this paper is to derive global necessary conditions satisfied by an optimal strategy, for the dynamic blocking problem introduced in [4]. As described in [4,5], these problems were originally motivated by the control of wild fires or the spatial spreading of a contaminating agent.

At each time $t \geq 0$, we denote by $R(t) \subset \mathbb{R}^{2}$ the region burned by the fire. In absence of control, for each $t \geq 0$ the set $R(t)$ is described as the reachable set for a differential inclusion:

$$
\dot{x} \in F(x) \quad x(0) \in R_{0},
$$

where the upper dot denotes a derivative w.r.t. time. In other words,

$$
R(t)=\left\{x(t) ; \quad x(\cdot) \text { absolutely continuous, } \quad x(0) \in R_{0}, \quad \dot{x}(\tau) \in F(x(\tau)) \text { for a.e. } \tau \in[0, t]\right\} .
$$

We assume that the initial set $R_{0} \subset \mathbb{R}^{2}$ is open and bounded. Moreover, we assume that $F: \mathbb{R}^{2} \mapsto \mathbb{R}^{2}$ is a Lipschitz continuous multifunction with compact, convex values, and satisfies

$$
0 \in F(x) \quad \text { for all } x \in \mathbb{R}^{2} \text {. }
$$

\footnotetext{
Keywords and phrases. Dynamic blocking problem, optimality conditions, differential inclusion with obstacles.

1 Department of Mathematics, Penn State University, University Park, Pa. 16802, USA. bressan@math.psu.edu; wang_t@math.psu.edu
} 
Clearly, this implies

$$
R\left(t_{1}\right) \subseteq R\left(t_{2}\right) \quad \text { whenever } t_{1}<t_{2} .
$$

In our model, the growth of the reachable set (i.e., the spreading of the fire) can be controlled by constructing barriers, in real time. Let $\psi: \mathbb{R}^{2} \mapsto R_{+}$be a continuous, strictly positive function. Calling $\gamma(t) \subset \mathbb{R}^{2}$ the portion of the wall constructed within time $t \geq 0$, we make the following assumptions:

(H1) For any $t_{1}<t_{2}$ one has $\gamma\left(t_{1}\right) \subseteq \gamma\left(t_{2}\right)$.

(H2) For every $t \geq 0$, the total length of the wall satisfies

$$
\int_{\gamma(t)} \psi \mathrm{d} m_{1} \leq t
$$

where $m_{1}$ denotes the one-dimensional Hausdorff measure, normalized so that $m_{1}(\Gamma)$ yields the usual length of a smooth curve $\Gamma$.

In the above formula, $1 / \psi(x)$ is the speed at which the wall can be constructed, at the location $x$. In particular, if $\psi(x) \equiv \sigma^{-1}$ is constant, then (1.4) simply means that the length of the curve $\gamma(t)$ is $\leq \sigma t$. A strategy $\gamma$ satisfying (H1)-(H2) will be called an admissible strategy. In addition, we say that the strategy $\gamma$ is complete if it satisfies:

(H3) For every $t \geq 0$ there holds

$$
\int_{\gamma(t)} \psi \mathrm{d} m_{1}=t, \quad \gamma(t)=\bigcap_{s>t} \gamma(s) .
$$

Moreover, if $\gamma(t)$ has positive upper density at a point $x$, i.e. if

$$
\limsup _{r \rightarrow 0+} \frac{m_{1}(B(x, r) \cap \gamma(t))}{r}>0,
$$

then $x \in \gamma(t)$. Here $B(x, r)$ is the open ball centered at $x$ with radius $r$.

As proved in [5], for every admissible strategy $t \mapsto \gamma(t)$ one can construct a second admissible strategy $t \mapsto \tilde{\gamma}(t) \supseteq \gamma(t)$, which is complete.

When a barrier is being constructed, the set reached by the fire is reduced. Namely, we define

$$
\begin{aligned}
R^{\gamma}(t) \doteq\left\{x(t) ; \quad x(\cdot) \text { absolutely continuous, } x(0) \in R_{0}\right. & \\
& \dot{x}(\tau) \in F(x(\tau)) \text { for a.e. } \tau \in[0, t], \quad x(\tau) \notin \gamma(\tau) \text { for all } \tau \in[0, t]\} .
\end{aligned}
$$

The existence of a blocking strategy, keeping $R^{\gamma}(t)$ uniformly bounded for all $t \geq 0$, was studied in [8,10].

To define an optimization problem, we need to introduce a cost functional. In general, this should take into account:

- The value of the area burned by the fire.

- The cost of building the barrier.

As in [4], we thus consider two continuous, non-negative functions $\alpha, \beta: \mathbb{R}^{2} \mapsto \mathbb{R}_{+}$and define the functional

$$
J(\gamma)=\int_{R_{\infty}^{\gamma}} \alpha \mathrm{d} m_{2}+\int_{\gamma_{\infty}} \beta \mathrm{d} m_{1},
$$


where the sets $R_{\infty}^{\gamma}, \gamma_{\infty}$ are defined respectively as

$$
R_{\infty}^{\gamma} \doteq \bigcup_{t \geq 0} R^{\gamma}(t), \quad \gamma_{\infty} \doteq \bigcup_{t \geq 0} \gamma(t)
$$

In (1.7), $m_{2}$ denotes the two-dimensional Lebesgue measure, while $m_{1}$ is the one-dimensional Hausdorff measure. In the case of a fire, $\alpha(x)$ is the value of a unit area of land at the point $x$, while $\beta(x)$ is the cost of building a unit length of wall at the point $x$. This leads to:

(OP1) Optimization Problem 1. Find an admissible strategy $t \mapsto \gamma(t)$ for which the corresponding functional $J(\gamma)$ at $(1.7)$ attains its minimum value.

For this problem, the existence of an optimal solution was proved in [5], under the following assumptions:

(A1) The initial set $R_{0}$ is open and bounded. Its boundary satisfies $m_{2}\left(\partial R_{0}\right)=0$.

(A2) The multifunction $F$ is Lipschitz continuous w.r.t. the Hausdorff distance. For each $x \in \mathbb{R}^{2}$ the set $F(x)$ is nonempty, closed and convex and contains the origin in its interior.

(A3) For every $x \in \mathbb{R}^{2}$ one has $\alpha(x) \geq 0, \beta(x) \geq 0, \alpha(x)+\beta(x)>0$, and $\psi(x) \geq \psi_{0}>0$. Moreover, $\alpha$ is locally integrable, while $\beta$ and $\psi$ are both lower semicontinuous.

In its original formulation, a strategy is a set-valued map $t \mapsto \gamma(t) \subset \mathbb{R}^{2}$ describing the portion of the wall constructed within a given time $t \geq 0$. The subsequent paper [9] showed that the above problem can be reformulated in a simpler way, where a strategy is entirely determined by assigning one single rectifiable set $\Gamma \subset \mathbb{R}^{2}$. We shall briefly review this equivalence result.

Consider a rectifiable set $\Gamma \subset \mathbb{R}^{2}$ which is complete, in the sense that it contains all of its points of positive upper density:

$$
\limsup _{r \rightarrow 0+} \frac{m_{1}(B(x, r) \cap \Gamma)}{r}>0 \quad \Longrightarrow \quad x \in \Gamma .
$$

Define the reachable set for the differential inclusion (1.1) restricted to $\mathbb{R}^{2} \backslash \Gamma$

$$
\begin{aligned}
& R^{\Gamma}(t) \doteq\left\{x(t) ; \quad x(\cdot) \text { absolutely continuous, } \quad x(0) \in R_{0},\right. \\
&\dot{x}(\tau) \in F(x(\tau)) \text { for a.e. } \tau \in[0, t], \quad x(\tau) \notin \Gamma \text { for all } \tau \in[0, t]\} .
\end{aligned}
$$

Throughout the following, $\bar{S}$ will denote the closure of a set $S$. We say that the rectifiable set $\Gamma$ is admissible in connection with the differential inclusion (1.1) and the bound on the construction speed (1.4) if

$$
\int_{\Gamma \cap \overline{R^{\Gamma}(t)}} \psi \mathrm{d} m_{1} \leq t \quad \text { for all } t \geq 0 .
$$

Of course, this means that the strategy

$$
t \mapsto \gamma(t) \doteq \Gamma \cap \overline{R^{\Gamma}(t)}
$$

is admissible according to (1.4). One can then consider: 
(OP2) Optimization Problem 2. Find an admissible rectifiable set $\Gamma \subset \mathbb{R}^{2}$ such that, calling $R_{\infty}^{\Gamma} \doteq$ $\bigcup_{t \geq 0} R^{\Gamma}(t)$, the cost

$$
J(\Gamma)=\int_{R_{\infty}^{\Gamma}} \alpha \mathrm{d} m_{2}+\int_{\Gamma} \beta \mathrm{d} m_{1}
$$

attains the minimum possible value.

As proved in [9], under the assumptions (A1)-(A3) the two formulations are equivalent. Namely, if $t \mapsto \gamma(t)$ is a complete, optimal strategy for (OP1), then the rectifiable set

$$
\Gamma \doteq\left(\bigcup_{t \geq 0} \gamma(t)\right) \backslash\left(\bigcup_{t \geq 0} R^{\gamma}(t)\right)
$$

is admissible and provides an optimal solution to the minimization problem (OP2). Vice versa, if the set $\Gamma$ provides an optimal solution to (OP2), then the strategy $\gamma(\cdot)$ in $(1.11)$ is optimal for (OP1).

Remark 1.1. For each $t \geq 0$, the set $\gamma(t)$ in (1.11) is the part of the wall $\Gamma$ touched by the fire at time $t$. This is the portion that actually needs to be put in place within time $t$, in order to constrain the fire. The remaining portion $\Gamma \backslash \gamma(t)$ can be constructed at a later time. On the other hand, given a strategy $\gamma(\cdot)$, the set $\Gamma$ consists of the "useful" part of all walls constructed by $\gamma$. Portions of a wall, which are constructed in a region already reached by the fire, are clearly useless.

Remark 1.2. By the assumption (A2), each velocity set $F(x)$ is a neighborhood of the origin. Hence the set $R_{\infty}^{\Gamma} \doteq \bigcup_{t \geq 0} R^{\Gamma}(t)$ of all points reached by the fire without crossing $\Gamma$ can be characterized as the union of all connected components of $\mathbb{R}^{2} \backslash \Gamma$ which intersect $R_{0}$.

Some necessary conditions for optimality were derived in [4], in the special case where $\beta \equiv 0$ and $\psi \equiv 1$, i.e. when there is no construction cost and the construction speed is constant. These conditions were essentially of local nature, obtained by perturbing the optimal strategy in a neighborhood of a given point.

The main goal of the present paper is to derive general optimality conditions, also of global nature. In particular, we study necessary conditions which must be satisfied at points of junction between two different arcs. We also analyze the case where the fire propagates along two or more fronts, and describe the optimal strategy at the time when one of these advancing fronts is extinguished.

As a preliminary, in Section 2 we introduce a concept of "regular strategy", and provide a careful classification of arcs. In particular, we observe that portions of an optimal barrier $\Gamma$ may be constructed not only to block the fire, but also to slow down its advancement. These will be called "delaying arcs". Their presence increases the time needed for the fire to reach some regions of the plane.

Sections 3 and 4 describe necessary conditions for the optimality of "free arcs", constructed away from the advancing fire front, and "boundary arcs", constructed right along the edge of an advancing front.

Section 6 deals with necessary conditions at junctions. In particular, we show that optimal arcs must join tangentially and we study the relations between Lagrange multipliers associated to different arcs.

Our analysis shows the existence of a scalar function $W(\cdot)$, which arises naturally as a global Lagrange multiplier, and can be interpreted as the "instantaneous value of time". Roughly speaking, $W(\tau)$ measures by how much the total cost could be reduced if the constraint (1.10) is replaced by

$$
\int_{\Gamma \cap \overline{R^{\Gamma}(t)}} \psi \mathrm{d} m_{1} \leq \begin{cases}t & \text { if } t<\tau \\ t+\varepsilon & \text { if } t \geq \tau\end{cases}
$$

The paper is concluded with two examples, where the optimal strategies and the value of time can be explicitly computed.

For the basic theory of differential inclusions and the minimum time function we refer to $[1,2]$. 


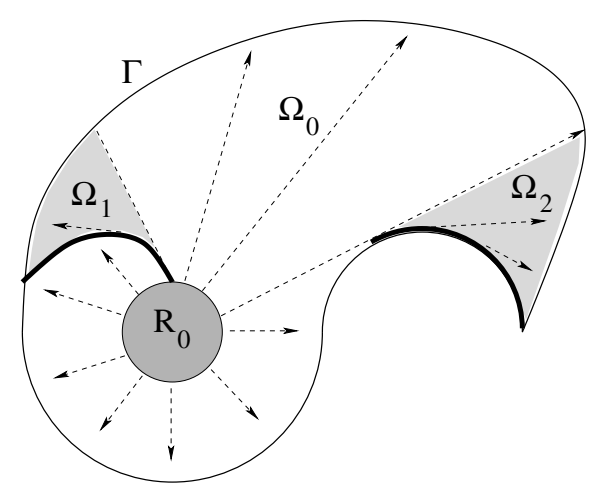

FIGURE 1. Here we take $R_{0}=F(x)=B(0,1)$, the unit disc centered at the origin. The two thick arcs denote the portion $\Gamma^{d} \subset \Gamma$ which contributes to slowing down the propagation of the fire. Notice that $T^{\Gamma}(x)=T(x)$ for $x \in \Omega_{0}$, but $T^{\Gamma}(x)>T(x)$ for $x \in \Omega_{1} \cup \Omega_{2}$. The thick arc next to the shaded region $\Omega_{1}$ lies in $\Gamma^{d} \backslash \Gamma^{b}$, the thick arc next to $\Omega_{2}$ lies in $\Gamma^{d} \cap \Gamma^{b}$.

\section{Regular Strategies AND CLASSifiCATION OF ARCS}

In this section, we introduce the basic framework and the regularity assumptions, in order to derive suitable necessary conditions for optimality.

Let $\Gamma$ be an admissible barrier for the differential inclusion (1.1), so that (1.10) holds. We observe that the construction of the barrier $\Gamma$ has two effects, namely: (i) it restricts the fire to the set $R_{\infty}^{\Gamma}$, consisting of all connected components of $\mathbb{R}^{2} \backslash \Gamma$ which intersect the initial domain $R_{0}$, and (ii) within the set $R_{\infty}^{\Gamma}$, it can slow down the advancement of the fire.

This fact, illustrated in Figure 1, can be better described as follows. Given the differential inclusion (1.1) and the barrier $\Gamma$, the minimum time function is defined as

$$
T^{\Gamma}(x) \doteq \inf \left\{t \geq 0 ; \quad x \in \overline{R^{\Gamma}(t)}\right\}
$$

Calling $T(x)$ the minimum time function for the problem (1.1) without any barrier, one clearly has

$$
0 \leq T(x) \leq T^{\Gamma}(x) \quad \text { for all } x \in \mathbb{R}^{2} .
$$

We say that a point $x \in \Gamma$ belongs to the delaying portion of the barrier if, by modifying the set $\Gamma$ in an arbitrarily small neighborhood of $x$, one can change the minimal time function somewhere else. More precisely, we introduce:

Definition 2.1. The subset $\Gamma^{d} \subseteq \Gamma$ of delaying walls is the set of all points $x \in \Gamma$ such that, for some $\delta>0$, the following holds. For every $\varepsilon>0$ there exists an admissible rectifiable set $\Gamma^{\prime}$ with $\Gamma^{\prime} \backslash B(x, \varepsilon)=\Gamma \backslash B(x, \varepsilon)$ and such that $T^{\Gamma^{\prime}}(y) \neq T^{\Gamma}(y)$ at some point $y \in R_{\infty}^{\Gamma} \backslash B(x, \delta)$.

We think of $\Gamma^{d}$ as a portion of the barrier $\Gamma$ which contributes to slowing down fire propagation. In addition, the barrier $\Gamma$ will contain an outer portion $\Gamma^{b}$, separating the burned from the unburned region.

Definition 2.2. The subset $\Gamma^{b} \subseteq \Gamma$ of blocking walls is defined as

$$
\Gamma^{b} \doteq \Gamma \cap \partial\left(\overline{R_{\infty}^{\Gamma}}\right)
$$

Remark 2.1. If $\Gamma$ is optimal, and the construction cost is strictly positive, then $\Gamma=\Gamma^{d} \cup \Gamma^{b}$. Indeed, any arc $\Gamma^{\prime} \subset \Gamma$ contained in the interior of the reachable set $R_{\infty}^{\Gamma}$ must be part of $\Gamma^{d}$. Otherwise the alternative strategy 
$\widetilde{\Gamma} \doteq \Gamma \backslash \Gamma^{\prime}$ would also be admissible, with a smaller cost. On the other hand, as shown in Figure 1, one can have $\Gamma^{d} \cap \Gamma^{b} \neq \emptyset$.

Given an admissible barrier $\Gamma$, a further classification of arcs can be achieved as follows. Define the set of times

$$
\mathcal{S} \doteq\left\{t \geq 0 ; \quad \int_{\Gamma \cap \overline{R^{\Gamma}(t)}} \psi \mathrm{d} m_{1}=t\right\}
$$

These are the times where the constraint is saturated, i.e. it is satisfied as an equality. We can further classify points $x \in \Gamma$ by setting

$$
\Gamma_{\mathcal{S}} \doteq\left\{x \in \Gamma ; \quad T^{\Gamma}(x) \in \mathcal{S}\right\}, \quad \Gamma_{\mathcal{F}} \doteq\left\{x \in \Gamma ; \quad T^{\Gamma}(x) \notin \mathcal{S}\right\} .
$$

As in [4], arcs lying in the subset $\Gamma_{\mathcal{F}}$ will be called free arcs.

A very general result on the existence of optimal blocking strategies was recently proved in [5]. However, this provides little information about the regularity of these optimal strategies. Namely, if $\Gamma$ is optimal, then $\Gamma$ must be the union of countably many compact, connected, rectifiable sets, plus a set whose 1-dimensional Hausdorff measure is zero. In order to derive necessary condition for optimality, additional regularity assumptions will be imposed.

Motivated by the definition of regular synthesis for an optimal control problem $[3,6,11,13]$, we consider a decomposition

$$
\mathbb{R}^{2}=\mathcal{M}_{1} \cup \cdots \cup \mathcal{M}_{M}
$$

with the following properties.

(i) Each $\mathcal{M}_{j} \subset \mathbb{R}^{2}$ is an embedded, connected $\mathcal{C}^{2}$ submanifold.

(ii) If $j \neq k$, then $\mathcal{M}_{j} \cap \mathcal{M}_{k}=\emptyset$.

(iii) If $\mathcal{M}_{j} \cap \overline{\mathcal{M}}_{k} \neq \emptyset$, then $\mathcal{M}_{j} \subset \overline{\mathcal{M}}_{k}$.

We call $d_{k} \doteq \operatorname{dim}\left(\mathcal{M}_{k}\right) \in\{0,1,2\}$, the dimension of the submanifold $\mathcal{M}_{k}$. In particular, $d_{k}=0$ if $\mathcal{M}_{k}$ consists of a single point and $d_{k}=2$ if $\mathcal{M}_{k}$ is an open subset of $\mathbb{R}^{2}$. In the case $d_{k}=1$, the above assumptions imply that $\mathcal{M}_{k}$ is a curve admitting a $\mathcal{C}^{2}$ parameterization in terms of arc-length.

Throughout the following, we assume that there exists a decomposition (2.4) such that the following holds.

(RA1) The barrier $\Gamma$ admits the decomposition

$$
\Gamma=\bigcup_{k \in \mathcal{B}} \mathcal{M}_{k}, \quad \text { for some } \quad \mathcal{B} \subset\{1,2, \ldots, M\} .
$$

Moreover, this decomposition is consistent with the previous classifications. Namely, each of the subsets $\Gamma^{d}, \Gamma^{b}, \Gamma_{S}, \Gamma_{\mathcal{F}}$ can be represented as a union of some of the manifolds $\mathcal{M}_{k}$.

(RA2) Restricted to each submanifold $\mathcal{M}_{j}$, the minimum time function $T^{\Gamma}$ is a $\mathcal{C}^{2}$ function, or else $T^{\Gamma} \equiv+\infty$.

Concerning the differential inclusion (1.1) we assume:

(RA3) The velocity sets $F(x)$ are uniformly convex, have $\mathcal{C}^{2}$ boundary, and contain the origin as an interior point. Moreover, denoting by $\langle\cdot, \cdot\rangle$ the Euclidean inner product, the map

$$
(p, x) \mapsto \arg \max _{y \in F(x)}\langle p, y\rangle
$$

is $\mathcal{C}^{2}$ on the set where $p \neq 0$. 
We observe that, away from the barrier $\Gamma$, the minimum time function $T^{\Gamma}$ is Lipschitz continuous and provides a viscosity solution to the Hamilton-Jacobi equation

$$
H(x, \nabla V)-1=0, \quad H(x, p) \doteq \max _{y \in F(x)}\langle p, y\rangle .
$$

We denote by

$$
h(x) \doteq \frac{1}{\left|\nabla T^{\Gamma}(x)\right|}=\max _{y \in F(x)}\langle\mathbf{n}(x), y\rangle \quad \mathbf{n}(x) \doteq \frac{\nabla T^{\Gamma}(x)}{\left|\nabla T^{\Gamma}(x)\right|},
$$

the propagation speed of the fire front, in the normal direction, at the point $x$.

Remark 2.2. The assumption (RA1) implies that each submanifold $\mathcal{M}_{k}$ with $k \in \mathcal{B}$ is a portion of the barrier $\Gamma$ falling in one single class of the above classification. For example, if $\mathcal{M}_{k}$ contains a point $x \in \Gamma_{\mathcal{S}}$, then $\mathcal{M}_{k} \subseteq \Gamma_{\mathcal{S}}$ and $\mathcal{M}_{k} \cap \Gamma_{\mathcal{F}}=\emptyset$.

Remark 2.3. If $\gamma \subset \Gamma_{\mathcal{F}} \backslash \Gamma^{d}$, then, as will be proved in the next section, any local perturbation of the arc $\gamma$ requiring the same construction time yields another admissible strategy. This is not true if $\gamma \subset \Gamma_{\mathcal{F}} \cap \Gamma^{d}$. Indeed, in this case a local perturbation of $\gamma$ will affect the minimum time function $T^{\Gamma}$ in other regions. Therefore, other $\operatorname{arcs} \tilde{\gamma} \subset \Gamma_{\mathcal{S}}$ may not be admissible any more.

Remark 2.4. Consider an arc $\gamma \subset \partial R^{\Gamma} \backslash \Gamma^{d}$. Then, the minimum time function $T^{\Gamma}$ can be extended from $R^{\Gamma}$ to a whole neighborhood of each point $x \in \Gamma$. Indeed, this extended value function $\widetilde{T}$ is constructed by solving the Hamilton-Jacobi equation (2.5) with data assigned along $\gamma$ :

$$
\max _{y \in F(x)} \nabla \widetilde{T}(x) \cdot y=1, \quad \widetilde{T}(x)=T^{\Gamma}(x) \quad \text { for } \quad x \in \gamma .
$$

In particular, the gradient $\nabla T^{\Gamma}(x)$ and the normal propagation speed $h(x)$ in (2.6) are well defined also for $x \in \gamma$, by a continuous extension.

\section{NECESSARY CONDITIONS FOR FREE ARCS}

In this section we consider an arc $\gamma \subset \Gamma_{\mathcal{F}}$. Intuitively, this means that at the time where this portion of wall is constructed, the fire has not yet reached points in $\gamma$. In addition, we assume that $\gamma \subseteq \Gamma^{b} \backslash \Gamma^{d}$. This means that $\gamma$ is a purely blocking arc. All minimum-time trajectories for the fire terminate when they reach a point of $\gamma$.

We seek necessary conditions for optimality of the arc $\gamma$. To fix the ideas, let $s \mapsto \gamma(s) \in \mathbb{R}^{2}, s \in[a, b]$, be a $\mathcal{C}^{2}$ parameterization of $\gamma$ in terms of arc-length. Throughout the sequel, an upper dot will denote a derivative w.r.t. $s$. Given two vectors $\mathbf{v}=\left(\begin{array}{l}a \\ b\end{array}\right)$ and $\mathbf{w}=\left(\begin{array}{l}c \\ d\end{array}\right)$, their wedge product will be written as $\mathbf{v} \wedge \mathbf{w} \doteq a d-b c$. By $\mathbf{t}(s) \doteq \dot{\gamma}(s)$ and $\mathbf{n}(s)$ we denote the unit vectors respectively tangent and perpendicular to the curve $\gamma$ at the point $\gamma(s)$, oriented so that $\mathbf{t} \wedge \mathbf{n}=1$.

We say that $\gamma$ is a normal arc if there exists a smooth scalar function $\varphi^{\sharp}:[a, b] \mapsto \mathbb{R}$ with $\varphi^{\sharp}(a)=\varphi^{\sharp}(b)=0$ such that, calling $\mathcal{T}\left(\gamma_{\varepsilon}^{\sharp}\right)$ the time needed to construct the arc $\gamma_{\varepsilon}^{\sharp}$ described by

$$
s \mapsto \gamma_{\varepsilon}^{\sharp}(s) \doteq \gamma(s)+\varepsilon \varphi^{\sharp}(s) \mathbf{n}(s),
$$

there holds

$$
\left[\frac{\mathrm{d}}{\mathrm{d} \varepsilon} \mathcal{T}\left(\gamma_{\varepsilon}^{\sharp}\right)\right]_{\varepsilon=0} \doteq\left[\frac{\mathrm{d}}{\mathrm{d} \varepsilon} \int_{a}^{b} \psi\left(\gamma_{\varepsilon}^{\sharp}(s)\right) \cdot\left|\dot{\gamma}_{\varepsilon}^{\sharp}(s)\right| \mathrm{d} s\right]_{\varepsilon=0}<0 .
$$

This implies that one can join the endpoints $P=\gamma(a)$ and $Q=\gamma(b)$ with some arc which can be constructed in a slightly shorter time: the curve $\gamma$ is not a time-minimizer. By possibly taking a small perturbation, it is not restrictive to assume that $\varphi^{\sharp}(s)=0$ for $s$ in a neighborhood of $a$ and $b$. 
Given a smooth function $\varphi:[a, b] \mapsto \mathbb{R}$ with $\varphi(s)=0$ for $s$ in a neighborhood of $a$ and $b$, and given $\varepsilon, \eta$ close to zero, consider the perturbed curve

$$
s \mapsto \gamma_{\varepsilon, \eta}(s) \doteq \gamma(s)+\left(\varepsilon \varphi(s)+\eta \varphi^{\sharp}(s)\right) \mathbf{n}(s) .
$$

By (3.2) and the implicit function theorem, for every $\varepsilon$ in a neighborhood of zero there exists a unique $\eta(\varepsilon)$ such that the time needed to construct the curves $\gamma$ and $\gamma_{\varepsilon, \eta(\varepsilon)}$ is the same. Calling $\gamma_{\varepsilon} \doteq \gamma_{\varepsilon, \eta(\varepsilon)}$, we thus have

$$
\left[\frac{\mathrm{d}}{\mathrm{d} \varepsilon} \mathcal{T}\left(\gamma_{\varepsilon}\right)\right]_{\varepsilon=0}=\frac{\mathrm{d}}{\mathrm{d} \varepsilon}\left[\int_{a}^{b} \psi\left(\gamma_{\varepsilon}(s)\right) \cdot\left|\dot{\gamma}_{\varepsilon}(s)\right| \mathrm{d} s\right]_{\varepsilon=0}=0 .
$$

We recall that $\dot{\mathbf{n}}(s)=\kappa(s) \mathbf{t}(s)$, where $\kappa(s)$ is the curvature of $\gamma$ at the point $\gamma(s)$. Since $|\dot{\gamma}| \equiv 1$, computing a derivative at $\varepsilon=0$ we find

$$
\left[\frac{\mathrm{d}}{\mathrm{d} \varepsilon}\left|\dot{\gamma}_{\varepsilon}\right|\right]_{\varepsilon=0}=\left\langle\dot{\gamma}, \frac{\mathrm{d}}{\mathrm{d} \varepsilon} \dot{\gamma}_{\varepsilon}\right\rangle_{\varepsilon=0}=\kappa(s)\left(\varphi(s)+\eta^{\prime}(0) \varphi^{\sharp}(s)\right) .
$$

The equation (3.4) can thus be written as

$$
\int_{a}^{b}\{\langle\nabla \psi(\gamma(s)), \mathbf{n}(s)\rangle+\psi(\gamma(s)) \kappa(\gamma(s))\} \cdot\left(\varphi(s)+\eta^{\prime}(0) \varphi^{\sharp}(s)\right) \mathrm{d} s=0 .
$$

For notational convenience, given a scalar function $g: \mathbb{R}^{n} \rightarrow \mathbb{R}$ we define

$$
\mathcal{G}(g, \gamma, s) \doteq\langle\nabla g(\gamma(s)), \mathbf{n}(s)\rangle+g(\gamma(s)) \kappa(s) .
$$

With this notation, from (3.5) it follows

$$
\eta^{\prime}(0)=-\frac{\int_{a}^{b} \mathcal{G}(\psi, \gamma, s) \varphi(s) \mathrm{d} s}{\int_{a}^{b} \mathcal{G}(\psi, \gamma, s) \varphi^{\sharp}(s) \mathrm{d} s} .
$$

Notice that in (3.7) the denominator is $\neq 0$, because by $(3.2)$

$$
\int_{a}^{b} \mathcal{G}(\psi, \gamma, s) \varphi^{\sharp}(s) \mathrm{d} s=\left[\frac{\mathrm{d}}{\mathrm{d} \varepsilon} \mathcal{T}\left(\gamma_{\varepsilon}^{\sharp}\right)\right]_{\varepsilon=0}<0 .
$$

Recalling that $\gamma \in \Gamma_{\mathcal{F}} \backslash \Gamma^{d}$, we now show that, for all $\varepsilon$ sufficiently close to zero, the barrier $\Gamma_{\varepsilon}$ obtained from $\Gamma$ replacing the arc $\gamma$ by $\gamma_{\varepsilon}$ is still admissible. To show this, we first observe that the map

$$
t \mapsto \int_{\Gamma \cap \overline{R^{\Gamma}(t)}} \psi \mathrm{d} m_{1}
$$

is non-decreasing and right-continuous, hence it is upper semicontinuous. In turn, the excess map

$$
\mathcal{E}(t) \doteq t-\int_{\Gamma \cap \frac{\Gamma}{R^{\Gamma}(t)}} \psi \mathrm{d} m_{1}
$$

is lower semicontinuous. 
To fix the ideas, assume $\left.T^{\Gamma}(\gamma(s)) \in\right] \tau_{0}, \tau[$ for $s \in] a, b[$, with $] \tau_{0}, \tau[\cap \mathcal{S}=\emptyset$. Moreover, assume that the perturbations $\varphi, \varphi^{\sharp}$ in (3.3) are supported in the compact subset $[a+\sigma, b-\sigma]$. By lower semicontinuity and compactness, there exists $\delta>0$ such that

$$
\mathcal{E}(T(\gamma(s)))>\delta \quad s \in[a+\sigma, b-\sigma] .
$$

Choose times $\tau_{0}<\tau_{1}<\ldots<\tau_{N}=\tau$ such that $\tau_{i}-\tau_{i-1}<\delta / 2$ for every $i=1, \ldots, N$. For each $i$, we can now choose $\varepsilon_{i}>0$ small enough such that, for $|\varepsilon| \leq \varepsilon_{i}$, there holds

$$
\int_{\Gamma_{\varepsilon} \cap \overline{R^{\Gamma \varepsilon}\left(\tau_{i}\right)}} \psi \mathrm{d} m_{1}<\frac{\delta}{2}+\int_{\Gamma \cap \overline{R^{\Gamma}\left(\tau_{i}\right)}} \psi \mathrm{d} m_{1}
$$

Setting $\bar{\varepsilon} \doteq \min \left\{\varepsilon_{1}, \ldots, \varepsilon_{N}\right\}$, we now prove that the barrier $\Gamma_{\varepsilon}$ is admissible whenever $|\varepsilon| \leq \bar{\varepsilon}$. Indeed, when $\tau_{i-1}<t \leq \tau_{i}$ we have

$$
\int_{\Gamma_{\varepsilon} \cap \overline{R^{\Gamma \varepsilon}(t)}} \psi \mathrm{d} m_{1} \leq \int_{\Gamma_{\varepsilon} \cap \overline{R^{\Gamma \varepsilon}\left(\tau_{i}\right)}} \psi \mathrm{d} m_{1}<\frac{\delta}{2}+\int_{\Gamma \cap \overline{R^{\Gamma}\left(\tau_{i}\right)}} \psi \mathrm{d} m_{1}=\frac{\delta}{2}+\tau_{i}-\mathcal{E}\left(\tau_{i}\right) \leq \tau_{i}-\frac{\delta}{2} \leq t .
$$

Next, call $J\left(\Gamma_{\varepsilon}\right)$ the total cost associated with this perturbed strategy. If $\Gamma$ is optimal, then

$$
\left[\frac{\mathrm{d}}{\mathrm{d} \varepsilon} J\left(\Gamma_{\varepsilon}\right)\right]_{\varepsilon=0}=0
$$

Assuming that the normal vector $\mathbf{n}$ points toward the outside of the burned region, by the previous analysis (3.10) can be written as

$$
\int_{a}^{b}\{\alpha(\gamma(s))+\mathcal{G}(\beta, \gamma, s)\} \cdot\left(\varphi(s)+\eta^{\prime}(0) \varphi^{\sharp}(s)\right) \mathrm{d} s=0 .
$$

Inserting the value of $\eta^{\prime}(0)$ given at (3.7), and adopting the shorter notation $\alpha(s) \doteq \alpha(\gamma(s))$, from the above equation we obtain

$$
\int_{a}^{b}\{\alpha(s)+\mathcal{G}(\beta, \gamma, s)\} \varphi(s) \mathrm{d} s+\lambda \cdot \int_{a}^{b} \mathcal{G}(\psi, \gamma, s) \varphi(s) \mathrm{d} s=0 .
$$

Here the constant $\lambda$ has the role of a Lagrange multiplier:

$$
\lambda=\frac{-\int_{a}^{b}\{\alpha(s)+\mathcal{G}(\beta, \gamma, s)\} \varphi^{\sharp}(s) \mathrm{d} s}{\int_{a}^{b} \mathcal{G}(\psi, \gamma, s) \varphi^{\sharp}(s) \mathrm{d} s} .
$$

Since (3.12) holds for all smooth functions $\varphi$ with $\varphi(a)=\varphi(b)=0$, recalling the definition of $\mathcal{G}(\cdot)$ we conclude

$$
\alpha(s)+\langle\nabla(\beta(s)+\lambda \psi(s)), \mathbf{n}(s)\rangle+(\beta(s)+\lambda \psi(s)) \kappa(s)=0
$$

for all $a<s<b$. Written as

$$
-(\beta(s)+\lambda \psi(s)) \kappa(s)=\alpha(\gamma(s))+\langle\nabla(\beta(s)+\lambda \psi(s)), \mathbf{n}(s)\rangle,
$$

this necessary condition takes the form of a second order nonlinear O.D.E., determining the curvature of $\gamma$. In the special case where $\psi$ and $\beta$ are constant, the above equation reduces to

$$
\kappa(s)=-\frac{\alpha(s)}{\beta+\lambda \psi},
$$



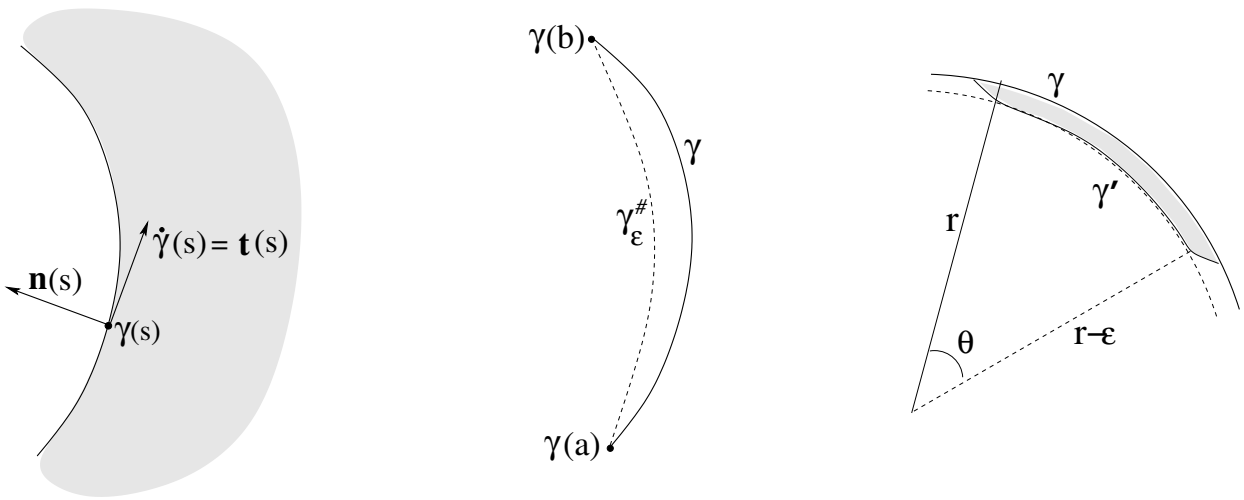

Figure 2. Left: The shaded region denotes the burned set. Since $\dot{\mathbf{n}}(s)=\kappa(s) \mathbf{t}(s)$, here the curvature $\kappa$ is negative. Center: For a normal extremal, one can find perturbations $\gamma_{\varepsilon}^{\sharp}$ that can be constructed in shorter time. Right: When a free arc $\gamma$ of radius $r$ is replaced by an arc $\gamma^{\prime}$ of smaller radius $r-\varepsilon$, the burned area increases by $\theta r \varepsilon$ while the length of the barrier decreases by $\theta \varepsilon$ (neglecting infinitesimals of higher order w.r.t. $\varepsilon$ ). If $\alpha, \beta$ in (1.7) satisfy $\beta>r \alpha$, then the total cost changes by $\alpha \theta r \varepsilon-\beta \theta \varepsilon<0$ and the arc $\gamma$ is not optimal.

showing that the curvature of $\gamma$ must be proportional to the local value of land $\alpha$. In particular, if $\alpha$ is also constant, then the free arc $\gamma$ is an arc of circumference.

Remark 3.1. The Lagrange multiplier $\lambda$ can be interpreted as the (constant, non-negative) value of time, during the construction of the arc $\gamma$. Indeed, given any smooth scalar function $\varphi:[a, b] \mapsto \mathbb{R}$ with $\varphi(a)=\varphi(b)=0$, for $\epsilon$ in a neighborhood of zero consider the perturbed arc $\tilde{\gamma}_{\epsilon}(s) \doteq \gamma(s)+\epsilon \varphi(s) \mathbf{n}(s)$. The time $\mathcal{T}\left(\tilde{\gamma}_{\epsilon}\right)$ needed to construct this arc satisfies

$$
\left[\frac{\mathrm{d}}{\mathrm{d} \epsilon} \mathcal{T}\left(\tilde{\gamma}_{\epsilon}\right)\right]_{\epsilon=0}=\int_{a}^{b} \mathcal{G}(\psi, \gamma, s) \varphi(s) \mathrm{d} s
$$

Calling $\Gamma_{\epsilon}$ the barrier obtained from $\Gamma$ by replacing the $\operatorname{arc} \gamma$ with $\tilde{\gamma}_{\epsilon}$, the cost $J\left(\Gamma_{\epsilon}\right)$ satisfies

$$
\left[\frac{\mathrm{d}}{\mathrm{d} \epsilon} J\left(\Gamma_{\epsilon}\right)\right]_{\epsilon=0}=\int_{a}^{b}\{\alpha(s)+\mathcal{G}(\beta, \gamma, s)\} \varphi(s) \mathrm{d} s .
$$

The ratio $\frac{[\text { decrease of the total cost }]}{\text { [increase in the construction time] }}$ now yields the value of time. Assuming that the quantity in (3.17) does not vanish, this ratio can be computed as

$$
\left[\frac{-\frac{\mathrm{d}}{\mathrm{d} \varepsilon} J\left(\Gamma_{\varepsilon}\right)}{\frac{\mathrm{d}}{\mathrm{d} \varepsilon} \mathcal{T}\left(\gamma_{\varepsilon}\right)}\right]_{\varepsilon=0}=\frac{-\int_{a}^{b}\{\alpha(s)+\mathcal{G}(\beta, \gamma, s)\} \varphi(s) \mathrm{d} s}{\int_{a}^{b} \mathcal{G}(\psi, \gamma, s) \varphi(s) \mathrm{d} s}=\lambda
$$

Indeed, by (3.13) and (3.12), the ratio does not depend on the choice of the function $\varphi$.

In the special case where the construction speed $\sigma=1 / \psi$ and the construction cost $\beta$ are constant, calling $r=-1 / \kappa$ the radius of curvature, from (3.16) it follows

$$
\lambda=(\alpha r-\beta) \sigma
$$

Notice that, in an optimal strategy, one must have $\alpha r-\beta \geq 0$. A geometric explanation of this inequality was provided in Figure 2. 
Next, we consider the case where $] \tau_{0}, \tau_{1}[\cap \mathcal{S}=\emptyset$, and the portion of wall constructed during this time interval consists of not just one but several free arcs, say

$$
\left\{x \in \Gamma ; \quad T^{\Gamma}(x) \in\right] \tau_{0}, \tau_{1}[\}=\gamma_{1} \cup \cdots \cup \gamma_{\nu} \subseteq \Gamma_{\mathcal{F}} \backslash \Gamma^{d} .
$$

Let the $i$ th arc be parameterized by arc-length, say $\left.s \mapsto \gamma_{i}(s), s \in\right] a_{i}, b_{i}[$. Assume that at least one of these arcs, say $\gamma_{1}$, is normal. Then we can find a compactly supported perturbation $\varphi_{1}^{\sharp}$ so that (3.2) holds.

Given a set of smooth perturbations with compact support $\left.\varphi_{i}:\right] a_{i}, b_{i}[\mapsto \mathbb{R}, i=1, \ldots, \nu$, for any $\varepsilon$ sufficiently close to zero we can find $\eta(\varepsilon)$ such that the total time needed to construct the $\nu$ perturbed curves

$$
\gamma_{1, \varepsilon}(s)=\gamma_{1}(s)+\left[\varepsilon \varphi_{1}(s)+\eta(\varepsilon) \varphi_{1}^{\sharp}(s)\right] \mathbf{n}_{1}(s), \quad \gamma_{i, \varepsilon}(s)=\gamma_{i}(s)+\varepsilon \varphi_{i}(s) \mathbf{n}_{i}(s) \quad i=2,3, \ldots, \nu,
$$

is the same for all $\varepsilon$. Hence

$$
\frac{\mathrm{d}}{\mathrm{d} \varepsilon} \sum_{i=1}^{\nu} \mathcal{T}\left(\gamma_{i, \varepsilon}\right)=\frac{\mathrm{d}}{\mathrm{d} \varepsilon}\left[\sum_{i=1}^{\nu} \int_{a_{i}}^{b_{i}} \psi\left(\gamma_{i, \varepsilon}(s)\right) \cdot\left|\dot{\gamma}_{i, \varepsilon}(s)\right| \mathrm{d} s\right] \equiv 0 .
$$

A similar argument as in (3.7) yields

$$
\eta^{\prime}(0)=-\sum_{i=1}^{\nu} \frac{\int_{a_{i}}^{b_{i}} \mathcal{G}\left(\psi, \gamma_{i}, s\right) \varphi_{i}(s) \mathrm{d} s}{\int_{a_{1}}^{b_{1}} \mathcal{G}\left(\psi, \gamma_{1}, s\right) \varphi_{1}^{\sharp}(s) \mathrm{d} s} .
$$

As before, one can show that the strategy $\Gamma_{\varepsilon}$ obtained by replacing each $\operatorname{arc} \gamma_{i}$ with $\gamma_{i, \varepsilon}$ is still admissible, as long as $\varepsilon$ remains sufficiently small. Since $\Gamma$ is optimal, the identity (3.10) must hold. In the present case, this yields

$$
\int_{a_{1}}^{b_{1}}\left(\alpha\left(\gamma_{1}(s)\right)+\mathcal{G}\left(\beta, \gamma_{1}, s\right)\right) \cdot \eta_{1}^{\prime}(0) \varphi_{1}^{\sharp}(s) \mathrm{d} s+\sum_{i=1}^{\nu} \int_{a_{i}}^{b_{i}}\left(\alpha\left(\gamma_{i}(s)\right)+\mathcal{G}\left(\beta, \gamma_{i}, s\right)\right) \varphi_{i}(s) \mathrm{d} s=0 .
$$

Hence there exists a Lagrange multiplier

$$
\lambda=-\frac{\int_{a_{1}}^{b_{1}}\left\{\alpha\left(\gamma_{1}(s)\right)+\mathcal{G}\left(\beta, \gamma_{1}, s\right)\right\} \varphi_{1}^{\sharp}(s) \mathrm{d} s}{\int_{a_{1}}^{b_{1}} \mathcal{G}\left(\psi, \gamma_{1}, s\right) \varphi_{1}^{\sharp}(s) \mathrm{d} s}
$$

such that

$$
\int_{a}^{b}\left\{\alpha\left(\gamma_{i}(s)\right)+\mathcal{G}\left(\beta, \gamma_{i}, s\right)\right\} \varphi_{i}(s) \mathrm{d} s+\lambda \cdot \int_{a}^{b} \mathcal{G}\left(\psi, \gamma_{i}, s\right) \varphi_{i}(s) \mathrm{d} s=0
$$

for all $i=1, \ldots, \nu$ and all perturbations $\varphi_{i}$ with compact support in $] a_{i}, b_{i}[$. As in (3.14)-(3.15), setting $\alpha_{i}(s) \doteq \alpha\left(\gamma_{i}(s)\right), \beta_{i}(s) \doteq \beta\left(\gamma_{i}(s)\right), \ldots$, and recalling the definition of $\mathcal{G}$, we conclude that

$$
\alpha_{i}(s)+\left(\left\langle\nabla\left(\beta_{i}(s)+\lambda \psi_{i}(s)\right), \mathbf{n}_{i}(s)\right\rangle+\left(\beta_{i}(s)+\lambda \psi_{i}(s)\right) \kappa_{i}(s)\right)=0
$$

for all $a_{i}<s<b_{i}$.

Summarizing the previous analysis, we now state a necessary condition for optimality, valid when several free arcs are simultaneously constructed.

Theorem 3.1 (necessary conditions for free arcs). Let $\gamma_{1}, \ldots, \gamma_{\nu} \subset \Gamma_{\mathcal{F}} \backslash \Gamma^{d}$ be free arcs, simultaneously constructed by an optimal strategy $\Gamma$ during the time interval $t \in] \tau_{0}, \tau_{1}[$. Assume that at least one of these arcs 
is normal, and let $s \mapsto \gamma_{i}(s)$ be a parameterization of $\gamma_{i}$ by arc-length, with $\left.s \in\right] a_{i}, b_{i}[$. Then there exists a Lagrange multiplier $\lambda \geq 0$ such that

$$
-\left(\beta_{i}(s)+\lambda \psi_{i}(s)\right) \kappa_{i}(s)=\alpha_{i}(s)+\left\langle\nabla\left(\beta_{i}(s)+\lambda \psi_{i}(s)\right), \mathbf{n}_{i}(s)\right\rangle
$$

for all $i=1, \ldots, \nu, \quad a_{i}<s<b_{i}$.

We recall that all curvatures $\kappa_{i}$ are negative, as explained in Figure 2.

As in Remark 3.1, the Lagrange multiplier $\lambda$ can be interpreted as the value of time. Note that this value is the same for all arcs $\gamma_{1}, \ldots, \gamma_{\nu}$, and remains constant throughout the time interval $] \tau_{0}, \tau_{1}[$ where the constraint (1.4) is unsaturated.

\section{NECESSARY CONDITIONS FOR BOUNDARY ARCS}

Let $\Gamma$ be an optimal barrier, and assume that for $t \in[a, b]$ the constraint (1.10) is saturated i.e. it is satisfied as an equality. To fix the ideas, assume that the subset

$$
\Gamma_{[a, b]} \doteq\left\{x \in \Gamma ; \quad a \leq T^{\Gamma}(x) \leq b\right\}
$$

consists of $\nu$ boundary $\operatorname{arcs} \gamma_{1}, \ldots, \gamma_{\nu} \subset \Gamma_{\mathcal{S}} \backslash \Gamma^{d}$, simultaneously constructed. Let each of these arcs be parameterized by time: $t \mapsto \gamma_{i}(t), t \in[a, b]$, so that $T^{\Gamma}\left(\gamma_{i}(t)\right)=t$ for each $i=1, \ldots, \nu$. We seek here necessary conditions for the optimality of these arcs. These will extend the conditions derived in [4] to the case where the cost functions $\alpha, \beta$ and the construction speed $1 / \psi$ are allowed to depend on the space variable $x$.

We say that $\gamma_{i}$ is a normal arc if, at every $t \in[a, b]$, the tangent vector $\dot{\gamma}_{i}(t)$ is not parallel to the gradient of the value function $\nabla T^{\Gamma}\left(\gamma_{i}(t)\right)$. Since $T^{\Gamma}\left(\gamma_{i}(t)\right)=t$ for all $t$, this is equivalent to the strict inequality

$$
\left|\dot{\gamma}_{i}(t)\right|>h(x) .
$$

In other words, the speed at which the $\operatorname{arc} \gamma_{i}$ is constructed is strictly greater than the local propagation speed $h(x)$ of the fire front, defined at (2.6). Throughout the following, we assume that all the $\operatorname{arcs} \gamma_{1}, \ldots, \gamma_{\nu}$ are normal.

We begin by choosing a suitable set of coordinates, around each arc $\gamma_{i}$. Let $\widetilde{T}$ be the minimum time function, extended to a neighborhood of each $\operatorname{arc} \gamma_{i}$, as in Remark 2.4. The assumption that the $\operatorname{arcs} \gamma_{i}$ are normal guarantees that these extensions are well defined. For $t \in[a, b]$ and $s$ close to zero, define a coordinate system $(t, s) \mapsto x_{i}(t, s)$ so that $x_{i}(t, 0)=\gamma_{i}(t)$, while, for each fixed time $t$, the map $s \mapsto x_{i}(t, s)$ provides an arc-length parameterization of the curve $\{x ; \widetilde{T}(x)=t\}$. To fix the ideas, we choose the orientation so that the points $x_{i}(t, s)$ with $s>0$ fall outside the set $R^{\Gamma}$ reached by the fire, as in Figure 3 . This implies

$$
\left\langle\mathbf{e}_{i}(t), \dot{\gamma}_{i}(t)\right\rangle<0
$$

where $\left.\mathbf{e}_{i}(t) \doteq \frac{\partial x_{i}(t, s)}{\partial s}\right|_{s=0}$ denotes the unit vector tangent to the curve $\{x ; \widetilde{T}(x)=t\}$ at the point $\gamma_{i}(t)$. In addition, we let $\mathbf{n}_{i}(t)$ be the unit vector parallel to $\nabla \widetilde{T}$ (hence perpendicular to $\mathbf{e}_{i}(t)$ ) at the point $\gamma_{i}(t)$, as in Figure 3.

Let $w_{i}^{*}(t)>0$ be the amount of resources allocated at time $t$ to the construction of the arc $\gamma_{i}$, so that

$$
\left|\dot{\gamma}_{i}(t)\right|=\frac{w_{i}^{*}(t)}{\psi\left(\gamma_{i}(t)\right)}, \quad \sum_{i=1}^{\nu} w_{i}^{*}(t) \equiv 1 \quad t \in[a, b] .
$$

Consider an alternative strategy $w=\left(w_{1}, \ldots, w_{\nu}\right)$. This will result in the construction of different $\operatorname{arcs} t \mapsto y_{i}(t)$, determined by the equations

$$
\left|\dot{y}_{i}(t)\right|=\frac{w_{i}(t)}{\psi\left(y_{i}(t)\right)}, \quad \widetilde{T}\left(y_{i}(t)\right)=t .
$$




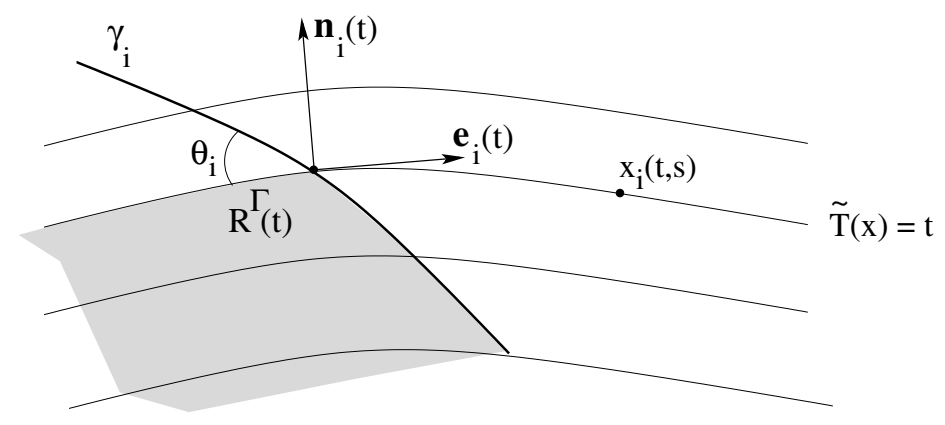

Figure 3. Choice of the coordinates $(t, s)$ in a neighborhood of the arc $\gamma_{i}$.

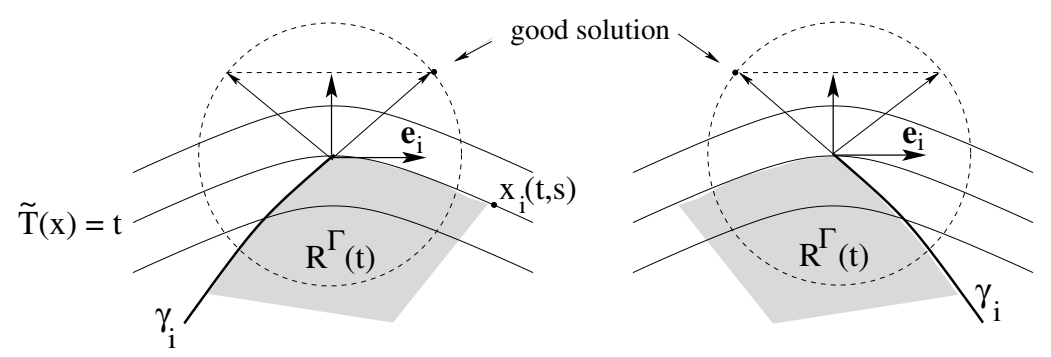

FiguRE 4. In equation (4.4), one should take $f_{i}$ as the larger or the smaller solution, respectively if the burned region lies to the right or to the left of the barrier $\gamma_{i}$.

Using our previous coordinate system, let $y_{i}(t)=x_{i}\left(t, s_{i}(t)\right)$. For each $i=1, \ldots, \nu$, the scalar function $s_{i}(t)$ will then satisfy an O.D.E. of the form

$$
\dot{s}_{i}=f_{i}\left(t, s_{i}(t), w_{i}(t)\right) .
$$

Here the right hand side $f_{i}$ is implicitly determined by the scalar constraint

$$
\left|\frac{\partial x_{i}\left(t, s_{i}\right)}{\partial t}+f_{i}\left(t, s_{i}, w_{i}\right) \frac{\partial x_{i}\left(t, s_{i}\right)}{\partial s_{i}}\right|=\frac{w_{i}}{\psi\left(x_{i}\left(t, s_{i}\right)\right)} .
$$

We observe that the equation (4.4) admits solutions provided that

$$
h\left(x_{i}\left(t, s_{i}\right)\right) \leq \frac{w_{i}}{\psi\left(x_{i}\left(t, s_{i}\right)\right)} .
$$

Indeed, the speed at which the barrier is constructed cannot be smaller than the propagation speed of the fire front, in the normal direction. In the case of a strict inequality, the equation (4.4) has exactly two solutions. The choice of the solution clearly depends on the side occupied by the burned region (see Fig. 4).

Assuming that the strategy $t \mapsto w^{*}(t)=\left(w_{1}^{*}, \ldots, w_{\nu}^{*}\right)(t)$ is optimal for the fire blocking problem, we now construct an auxiliary control problem for which $w^{*}$ is optimal as well. Consider the control system consisting of the $\nu$ equations (4.3), supplemented by the initial and terminal constraints,

$$
s_{i}(a)=s_{i}(b)=0 \quad i=1, \ldots, \nu
$$

Calling

$$
\mathbb{R}_{+}^{\nu} \doteq\left\{w=\left(w_{1}, \ldots, w_{\nu}\right) ; \quad w_{i} \geq 0 \quad \text { for all } i=1, \ldots, \nu\right\}
$$


the family of admissible control functions is defined as

$$
\mathcal{W} \doteq\left\{w:[a, b] \mapsto \mathbb{R}_{+}^{\nu} ; w \text { measurable, } \quad \sum_{i=1}^{\nu} \int_{a}^{t} w_{i}(\tau) \mathrm{d} \tau \leq t-a \quad \text { for all } t \in[a, b]\right\} .
$$

Now consider the optimization problem

$$
\text { minimize: } \Lambda(w) \doteq \sum_{i=1}^{\nu} \int_{a}^{b} L_{i}\left(t, s_{i}(t), w_{i}(t)\right) \mathrm{d} t,
$$

where the running costs are

$$
L_{i}\left(t, s_{i}, w_{i}\right) \doteq \beta\left(x_{i}\left(t, s_{i}\right)\right) \frac{w_{i}(t)}{\psi\left(x_{i}\left(t, s_{i}\right)\right)}+\int_{0}^{s_{i}} h\left(x_{i}(t, \xi)\right) \alpha\left(x_{i}(t, \xi)\right) \mathrm{d} \xi,
$$

with $h(\cdot)$ as in definition (2.6). The minimum in (4.8) is sought among all control functions $w \in \mathcal{W}$. Notice that the first term in (4.9) accounts for the cost of building the wall, while the second term is related to the value of the burned area. We are assuming here that the burned region has the representation $\left\{x_{i}(t, s) ; s<s_{i}(t)\right\}$.

It is convenient to introduce two additional state variables, to account for the cost functional (4.8) and for the integral constraint in (4.7), which will be reformulated as a pointwise state constraint. We thus consider the variables $s_{0}, s_{\nu+1}$, governed by the equations

$$
\begin{gathered}
s_{0}(a)=0, \quad \dot{s}_{0}(t)=f_{0}(w(t)) \doteq-1+\sum_{i=1}^{\nu} w_{i}(t), \\
s_{\nu+1}(a)=0, \quad \dot{s}_{\nu+1}(t)=f_{\nu+1}(t, s(t), w(t)) \doteq \sum_{i=1}^{\nu} L_{i}\left(t, s_{i}(t), w_{i}(t)\right) .
\end{gathered}
$$

For a system with state variables $s=\left(s_{0}, s_{1}, \ldots, s_{\nu+1}\right)$ and dynamics $(4.3),(4.6),(4.10),(4.11)$, we now consider the optimization problem

$$
\text { minimize: } s_{\nu+1}(b)
$$

with state constraint

$$
s_{0}(t) \leq 0 \quad \text { for all } t \in[a, b] .
$$

The minimum is sought among all measurable controls $w=\left(w_{1}, \ldots, w_{\nu}\right):[a, b] \mapsto \mathbb{R}_{+}^{\nu}$.

By construction, the control $w^{*}(t)=\left(w_{1}^{*}(t), \ldots, w_{\nu}^{*}(t)\right)$ corresponding to the trajectory $t \mapsto\left(s_{0}(t), s_{1}(t), \ldots\right.$, $\left.s_{\nu}(t)\right) \equiv(0,0, \ldots, 0)$ is optimal for this auxiliary optimal control problem. We recall that

$$
w_{i}^{*}(t)>0, \quad \sum_{i=1}^{\nu} w_{i}^{*}(t)=1 \quad t \in[a, b] .
$$

Using a version of the Pontryagin maximum principle in the presence of the state constraints (see [12,15]), we conclude that there exists $\lambda_{0} \geq 0$, and a map $t \mapsto q(t)=\left(q_{0}(t), \ldots, q_{\nu}(t)\right)$, not both equal to zero, such that the following holds. The map $q_{0}$ satisfies

$$
q_{0}(b)=0, \quad q_{0}(t)=q_{0}(a)-\int_{a}^{t} \mathrm{~d} \mu
$$

where $\mu$ is any positive measure supported on the set where $s_{0}=0$. Since by assumption this set is the entire interval $[a, b]$, this is equivalent to

$$
t \mapsto q_{0}(t) \quad \text { is bounded, non-increasing, } \quad q_{0}(b)=0 .
$$


Moreover, the other components $q_{1}, \ldots, q_{\nu}$ are absolutely continuous functions such that

$$
\dot{q}_{i}(t)=-q_{i} \frac{\partial f_{i}}{\partial s_{i}}\left(t, 0, w_{i}^{*}(t)\right)-\lambda_{0} \frac{\partial L_{i}}{\partial s_{i}}\left(t, 0, w_{i}^{*}(t)\right) \quad i=1, \ldots, \nu
$$

for a.e. $t \in[a, b]$. Finally, for a.e. $t \in[a, b]$ there holds

$$
\begin{aligned}
q_{0}(t)+\lambda_{0} \sum_{i=1}^{\nu} L_{i}\left(t, 0, w_{i}^{*}(t)\right)+\sum_{i=1}^{\nu} & q_{i}(t) f_{i}\left(t, 0, w_{i}^{*}(t)\right) \\
& =\min _{w \in \mathbb{R}_{+}^{\nu}}\left(q_{0}(t) \sum_{i=1}^{\nu} w_{i}+\lambda_{0} \sum_{i=1}^{\nu} L_{i}\left(t, 0, w_{i}\right)+\sum_{i=1}^{\nu} q_{i}(t) f_{i}\left(t, 0, w_{i}\right)\right) .
\end{aligned}
$$

Differentiating w.r.t. $w_{1}, \ldots, w_{\nu}$, from (4.16) we deduce

$$
-q_{i}(t) \frac{\partial f_{i}}{\partial w_{i}}\left(t, 0, w_{i}^{*}(t)\right)-\lambda_{0} \frac{\partial L_{i}}{\partial w_{i}}\left(t, 0, w_{i}^{*}(t)\right)=q_{0}(t) \quad i=1, \ldots, \nu .
$$

We now work out a more explicit form of the equations (4.15) and of the conditions (4.17). From the definition of $L_{i}$ at (4.9) it follows

$$
\frac{\partial L_{i}}{\partial s_{i}}\left(t, 0, w_{i}^{*}(t)\right)=\left\langle\nabla\left(\frac{\beta}{\psi}\right)\left(\gamma_{i}(t)\right), \mathbf{e}_{i}(t)\right\rangle w_{i}^{*}(t)+h_{i}\left(\gamma_{i}(t)\right) \alpha\left(\gamma_{i}(t)\right) .
$$

Toward the computation of $\partial f_{i} / \partial s_{i}$, consider a family of perturbed trajectories of the form

$$
t \rightarrow \gamma_{i}^{\varepsilon}(t)=\gamma_{i}\left(t, \varepsilon \zeta(t)+\mathcal{O}\left(\varepsilon^{2}\right)\right)=\gamma_{i}(t)+\varepsilon \zeta(t) \mathbf{e}_{i}(t)+\mathcal{O}\left(\varepsilon^{2}\right),
$$

such that for all $\varepsilon, t$

$$
\left|\dot{\gamma}_{i}^{\varepsilon}(t)\right| \cdot \psi\left(\gamma_{i}^{\varepsilon}(t)\right)=\left|\dot{\gamma}_{i}(t)\right| \cdot \psi\left(\gamma_{i}(t)\right)=w_{i}^{*}(t)
$$

In particular, the above identities imply

$$
\frac{\mathrm{d}}{\mathrm{d} \varepsilon}\left[\left|\dot{\gamma}_{i}^{\varepsilon}(t)\right|^{2} \psi^{2}\left(\gamma_{i}^{\varepsilon}(t)\right)\right]_{\varepsilon=0}=0 .
$$

Using (4.19) in the above equation, we obtain

$$
\left\langle\dot{\gamma}_{i}(t), \dot{\zeta}(t) \mathbf{e}_{i}(t)+\zeta(t) \dot{\mathbf{e}}_{i}(t)\right\rangle \psi\left(\gamma_{i}(t)\right)+\left\langle\nabla \psi\left(\gamma_{i}(t)\right), \zeta(t) \mathbf{e}_{i}(t)\right\rangle\left|\dot{\gamma}_{i}(t)\right|^{2}=0 .
$$

Solving (4.21) for $\dot{\zeta}$ and recalling (4.20), we derive the first order linear O.D.E.:

$$
\dot{\zeta}=-\frac{\left\langle\dot{\gamma}_{i}(t), \dot{\mathbf{e}}_{i}(t)\right\rangle \psi\left(\gamma_{i}(t)\right)+\left\langle\nabla \psi\left(\gamma_{i}(t)\right), \mathbf{e}_{i}(t)\right\rangle\left|\dot{\gamma}_{i}(t)\right|^{2}}{\left\langle\dot{\gamma}_{i}(t), \mathbf{e}_{i}(t)\right\rangle \psi\left(\gamma_{i}(t)\right)} \zeta .
$$

On the other hand, we observe that the scalar functions $s_{i}^{\varepsilon}=\varepsilon \zeta(t)+\mathcal{O}\left(\varepsilon^{2}\right)$ in (4.19) are solutions to the same O.D.E.

$$
\dot{s}_{i}^{\varepsilon}(t)=f_{i}\left(t, s_{i}^{\varepsilon}(t), w_{i}^{*}(t)\right)
$$

with possibly different initial data. Hence the first order term $\zeta(\cdot)$ in the expansion provides a solution to the linear equation

$$
\dot{\zeta}=\frac{\partial f_{i}}{\partial s_{i}}\left(t, 0, w_{i}^{*}(t)\right) \zeta
$$


Comparing (4.22) with (4.23) we conclude

$$
\frac{\partial f_{i}}{\partial s_{i}}\left(t, 0, w_{i}^{*}(t)\right)=-\frac{\left\langle\dot{\gamma}_{i}(t), \dot{\mathbf{e}}_{i}(t)\right\rangle \psi\left(\gamma_{i}(t)\right)+\left\langle\nabla \psi\left(\gamma_{i}(t)\right), \mathbf{e}_{i}(t)\right\rangle\left|\dot{\gamma}_{i}(t)\right|^{2}}{\left\langle\dot{\gamma}_{i}(t), \mathbf{e}_{i}(t)\right\rangle \psi\left(\gamma_{i}(t)\right)} .
$$

The equations (4.18) and (4.24) provide a more explicit expression of the right hand side of (4.15).

To compute the partial derivative $\partial f_{i} / \partial w_{i}$ at points $\left(t, 0, w_{i}^{*}(t)\right)$, we start by writing (4.4) in the equivalent form

$$
\left|\frac{\partial x_{i}\left(t, s_{i}\right)}{\partial t}+f_{i}\left(t, s_{i}, w_{i}\right) \frac{\partial x_{i}\left(t, s_{i}\right)}{\partial s_{i}}\right|^{2}=\left(\frac{w_{i}}{\psi\left(x_{i}\left(t, s_{i}\right)\right)}\right)^{2} .
$$

Since our choice of coordinates $\left(t, s_{i}\right)$ implies $\frac{\partial x_{i}}{\partial s_{i}}(t, 0)=\mathbf{e}_{i}(t)$ and $f_{i}\left(t, 0, w_{i}^{*}(t)\right) \equiv 0$, differentiating (4.25) w.r.t. $w_{i}$ we obtain

$$
\left\langle\dot{\gamma}_{i}(t), \mathbf{e}_{i}(t)\right\rangle \frac{\partial f_{i}}{\partial w_{i}}\left(t, 0, w_{i}^{*}(t)\right)=\frac{w_{i}^{*}(t)}{\psi^{2}\left(\gamma_{i}(t)\right)} .
$$

For convenience, we denote by $\theta_{i}(t)$ the angle between the barrier $\gamma$ and the level set $\{\tilde{T}(x)=t\}$, as in Figure 3 . Notice that this implies

$$
\left\langle\frac{\dot{\gamma}_{i}(t)}{\left|\dot{\gamma}_{i}(t)\right|}, \mathbf{e}_{i}(t)\right\rangle=-\cos \theta_{i}(t)
$$

Recalling that the optimal control is $w_{i}^{*}(t)=\left|\dot{\gamma}_{i}(t)\right| \cdot \psi\left(\gamma_{i}(t)\right)$, from the identity (4.26) we deduce

$$
\frac{\partial f_{i}}{\partial w_{i}}\left(t, 0, w_{i}^{*}(t)\right)=-\frac{1}{\psi\left(\gamma_{i}(t)\right)} \cdot \frac{1}{\cos \theta_{i}(t)}<0
$$

We observe that the map $w_{i} \mapsto f_{i}\left(t, 0, w_{i}\right)$ is well defined and monotone decreasing, for $w_{i}$ in a neighborhood of $w_{i}^{*}(t)$. Intuitively, if we increase the amount $w_{i}$ of resources allocated to the construction of the barrier $\gamma_{i}$, then the construction speed $|\dot{\gamma}|$ increases. As a result, the angle $\theta_{i}$ decreases and the barrier will be shifted toward the left, further reducing the region burned by the fire (see Fig. 3).

We also notice that (4.14) implies that $q_{0}(t) \geq 0$ for all $t \in[a, b]$. Summarizing the above arguments, we now state a set of necessary conditions for the optimality of multiple arcs which are constructed simultaneously. Recall that $f_{i}, L_{i}$ are the functions in (4.3) and (4.9), while $\theta_{i}$ is the angle between the barrier and the fire front.

Theorem 4.1 (necessary conditions for boundary arcs). Let $\gamma_{1}, \ldots, \gamma_{\nu} \subset \Gamma_{\mathcal{S}} \backslash \Gamma^{d}$ be the boundary arcs simultaneously constructed by an optimal strategy $\Gamma$, during the time interval $t \in\left[\tau_{1}, \tau_{2}\right]$. Assume that each arc $t \mapsto \gamma_{i}(t)$ is normal, parameterized by time $t=T^{\Gamma}\left(\gamma_{i}(t)\right)$, and call $\mathbf{e}_{i}(t)$ the unit vector tangent to the boundary of the reachable set $R^{\Gamma}(t)$ at the point $\gamma_{i}(t)$, oriented toward the outside of the reachable set. Then there exist a constant $\lambda_{0} \geq 0$, and an adjoint vector $q=\left(q_{0}, q_{1}, \ldots, q_{\nu}\right)$, not both equal to zero, satisfying the following conditions. The map $t \mapsto q_{0}(t)$ is non-negative and non increasing, while for $i=1, \ldots, \nu$ the map $t \mapsto q_{i}(t)$ is absolutely continuous and satisfies

$$
\begin{aligned}
\dot{q}_{i}(t)= & q_{i}(t) \cdot \frac{\left\langle\dot{\gamma}_{i}(t), \dot{\mathbf{e}}_{i}(t)\right\rangle \psi\left(\gamma_{i}(t)\right)+\left\langle\nabla \psi\left(\gamma_{i}(t)\right), \mathbf{e}_{i}(t)\right\rangle\left|\dot{\gamma}_{i}(t)\right|^{2}}{\left\langle\dot{\gamma}_{i}(t), \mathbf{e}_{i}(t)\right\rangle \psi\left(\gamma_{i}(t)\right)} \\
& -\lambda_{0}\left[\left\langle\nabla\left(\frac{\beta}{\psi}\right)\left(\gamma_{i}(t)\right), \mathbf{e}_{i}(t)\right\rangle w_{i}^{*}(t)+h_{i}\left(\gamma_{i}(t)\right) \alpha\left(\gamma_{i}(t)\right)\right] .
\end{aligned}
$$

The optimality conditions

$$
q_{0}(t) w_{i}^{*}(t)+\lambda_{0} L_{i}\left(t, 0, w_{i}^{*}(t)\right)+q_{i}(t) f_{i}\left(t, 0, w_{i}^{*}(t)\right)=\min _{\omega \geq 0}\left\{q_{0}(t) \omega+\lambda_{0} L_{i}(t, 0, \omega)+q_{i}(t) f_{i}(t, 0, \omega)\right\}
$$


hold for every $i=1, \ldots, \nu$ and $\tau_{1}<t<\tau_{2}$. Moreover, the functions

$$
W_{i}(t) \doteq \frac{1}{\cos \theta_{i}(t)} \frac{q_{i}(t)}{\psi\left(\gamma_{i}(t)\right)}-\lambda_{0} \cdot \frac{\beta\left(\gamma_{i}(t)\right)}{\psi\left(\gamma_{i}(t)\right)} \quad i=1, \ldots, \nu
$$

are non-negative, non-increasing, and all equal to each other.

Indeed, the first part of the theorem is a reformulation of the Pontryagin maximum principle. Concerning the last statement, recalling (4.27), (4.9), and the above definition of $W_{i}$, by (4.17) we conclude

$$
W_{i}(t)=-q_{i}(t) \frac{\partial f_{i}}{\partial w_{i}}\left(t, 0, w_{i}^{*}(t)\right)-\lambda_{0} \frac{\partial L_{i}}{\partial w_{i}}\left(t, 0, w_{i}^{*}(t)\right)=q_{0}(t) \quad i=1, \ldots, \nu
$$

\section{NECESSARY CONDITIONS AT JUNCTIONS}

The necessary conditions for optimality derived in the previous two sections were of local nature. Indeed, we always used perturbations of free arcs or of boundary arcs which kept the endpoints fixed. In this section, we shall obtain stronger optimality conditions, of global nature, by allowing changes also at the endpoints of the various arcs.

We recall that, for a free arc $\gamma$, the Lagrange multiplier $\lambda$ introduced at (3.19) could be interpreted as the value of time, which is constant during the interval when the free arc is constructed. If the construction cost $\beta$ and the construction speed $\sigma=1 / \psi$ are constant, then by (3.20) this value of time is computed as

$$
W(t)=(\alpha r-\beta) \sigma
$$

Here $\alpha$ is the unit value of the land, while $r$ is the radius of curvature of the barrier $\gamma$. According to Theorem 3.1, if several free $\operatorname{arcs} \gamma_{1}, \ldots, \gamma_{\nu}$ are simultaneously constructed, the values $\left(\alpha\left(\gamma_{i}(x)\right) r_{i}(x)-\beta\right) \sigma$ are all equal to each other.

On the other hand, when boundary arcs are constructed, the functions $W_{i}(t)$ in $(4.30)$ are defined only up to a positive constant. Indeed, they depend on the choice of the adjoint variables $q_{i}, \lambda_{0}$. In the present section we consider some particular configurations of optimal barriers, where one can take $\lambda_{0}=1$ and let $\left(q_{1}, \ldots, q_{\nu}\right)=\nabla V$ be the gradient of a value function. In this case, the instantaneous value of time is well defined as

$$
W(t)=W_{i}(t)=\frac{1}{\cos \theta_{i}(t)} \frac{q_{i}(t)}{\psi\left(\gamma_{i}(t)\right)}-\frac{\beta\left(\gamma_{i}(t)\right)}{\psi\left(\gamma_{i}(t)\right)} \quad i=1, \ldots, \nu
$$

\subsection{Two boundary arcs joining together}

We start by examining the case where two boundary arcs $\gamma_{1}, \gamma_{2}$ join together at the terminal point $P$ at time $T$, thus completing the wall construction, as in Figure 5.

We show that this situation can be modelled by an optimal control system in standard form, with free terminal time. Indeed, let $(t, s) \mapsto x(t, s)$ be a system of coordinates, chosen so that:

- for each fixed $t$, the map $s \mapsto x(t, s)$ is an arc-length parameterization of the boundary $\partial R^{\Gamma}(t)$, so that

$$
T^{\Gamma}(x(t, s))=t, \quad\left|\frac{\partial}{\partial s} x(t, s)\right| \equiv 1 .
$$

We introduce an auxiliary optimal control problem, with state $s=\left(s_{0}, s_{1}, s_{2}\right)$ and control variable $w=\left(w_{1}, w_{2}\right)$.

$$
\text { Minimize: } \quad s_{0}(T)
$$



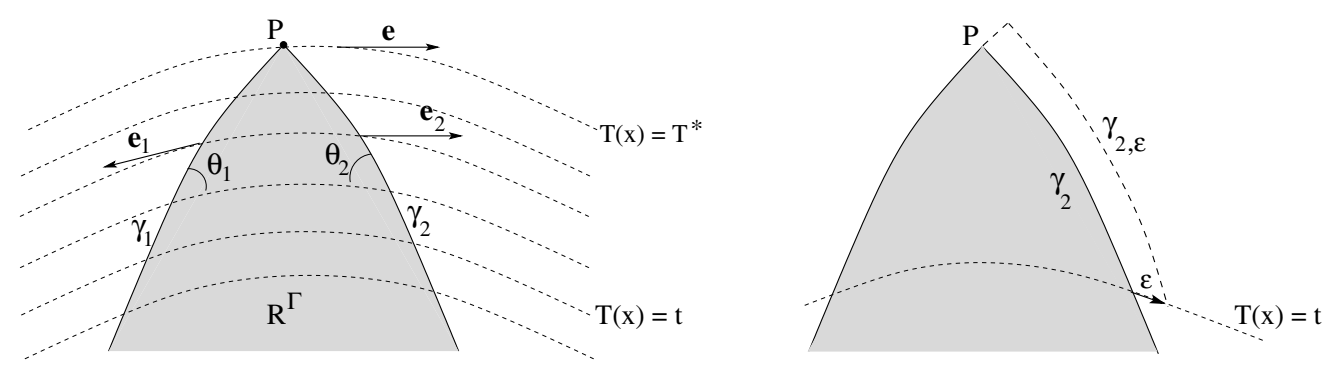

Figure 5. Left: two boundary arcs joining at the terminal point. Right: if the construction is completed as the two walls meet at $P$, one can consider the perturbed problem, where at time $t$ the position of the wall $\gamma_{2}$ is shifted by $\varepsilon$ along the fire front $\{x ; T(x)=t\}$. This yields a slightly different $\operatorname{cost} J_{\varepsilon}$. One can then uniquely determine the adjoint variable by setting $q_{2}(t)=\left[\frac{d}{d \varepsilon} J_{\varepsilon}\right]_{\varepsilon=0}$.

for the system with dynamics

$$
\left\{\begin{array}{l}
\dot{s}_{0}=f_{0}\left(t, s_{1}, s_{2}, w_{1}, w_{2}\right) \\
\dot{s}_{1}=f_{1}\left(t, s_{1}, w_{1}\right) \\
\dot{s}_{2}=f_{2}\left(t, s_{2}, w_{2}\right)
\end{array}\right.
$$

and terminal constraints

$$
s_{1}(T)-s_{2}(T)=0 .
$$

Here the controls $w_{1}, w_{2}$ satisfy the constraints

$$
w_{i}(t) \in[0,1], \quad w_{1}(t)+w_{2}(t) \leq 1
$$

Moreover,

$$
\begin{aligned}
f_{0}\left(t, s_{1}, s_{2}, w_{1}, w_{2}\right) \doteq & \beta\left(x\left(t, s_{1}(t)\right)\right) \frac{w_{1}(t)}{\psi\left(x\left(t, s_{1}(t)\right)\right)}+\beta\left(x\left(t, s_{2}(t)\right)\right) \frac{w_{2}(t)}{\psi\left(x\left(t, s_{2}(t)\right)\right)} \\
& +\int_{s_{1}(t)}^{s_{2}(t)} h(x(t, \xi)) \alpha(x(t, \xi)) \mathrm{d} \xi
\end{aligned}
$$

The first two terms in the definition of $f_{0}$ account for the cost of building the two walls, while the integral term keeps track of the increase in the burned area. As in $(2.6), h(x)$ is the normal velocity of the advancing fire front, at the point $x$. The functions $f_{1}, f_{2}$ are implicitly determined by the identities

$$
\left|\frac{\partial x\left(t, s_{i}\right)}{\partial t}+f_{i}\left(t, s_{i}, w_{i}\right) \cdot \frac{\partial x\left(t, s_{i}\right)}{\partial s_{i}}\right|=\frac{w_{i}}{\psi\left(x\left(t, s_{i}\right)\right)} \quad i=1,2 .
$$

As remarked in the previous section, each equation in (5.8) has two solutions. If the orientation of the vector $\mathbf{e}=\frac{\partial x(t, s)}{\partial s}$ is as shown in Figure 5, one should choose the larger solution for $i=1$ and the smaller solution for $i=2$.

Let $t \mapsto w^{*}(t)=\left(w_{1}^{*}, w_{2}^{*}\right)(t)$ be an optimal control, and let $t \mapsto s^{*}(t)=\left(s_{1}^{*}, s_{2}^{*}\right)(t)$ be the corresponding optimal trajectory. Then, by the Pontryagin maximum principle [7], there exists an absolutely continuous 
adjoint vector $p(t)=\left(p_{0}, p_{1}, p_{2}\right)(t)$ such that the following holds:

$$
\begin{gathered}
\dot{p}_{0} \equiv 0, \quad \dot{p}_{i}=-\sum_{k=0}^{2} \frac{\partial f_{k}}{\partial s_{i}} p_{k} \quad i=1,2, \\
\sum_{i=0}^{2} p_{i}(t) \cdot f_{i}\left(t, s^{*}(t), w^{*}(t)\right)=\min _{w_{1}+w_{2} \leq 1} \sum_{i=0}^{2} p_{i}(t) \cdot f_{i}\left(t, s^{*}(t), w_{i}\right)
\end{gathered}
$$

at almost every time $t$. Assuming that both arcs are normal, as in (4.1), we can here normalize the adjoint vector by taking $p_{0} \equiv 1$. By (5.5), at the terminal time $t=T^{*}$ one has

$$
\left(p_{0}, p_{1}, p_{2}\right)\left(T^{*}\right)=(1,-\eta, \eta)
$$

for some real number $\eta$. This Lagrange multiplier can be determined using the further relation

$$
\min _{w_{1}+w_{2} \leq 1}\left\{f_{0}\left(T^{*}, s^{*}\left(T^{*}\right), w_{1}, w_{2}\right)-\eta f_{1}\left(T^{*}, s_{1}^{*}\left(T^{*}\right), w_{1}\right)+\eta f_{2}\left(T^{*}, s_{2}^{*}\left(T^{*}\right), w_{2}\right)\right\}=0 .
$$

By the first equation in (5.9) and the terminal conditions (5.11) it follows $p_{0}(t) \equiv 1$. Setting

$$
\xi_{i}(t) \doteq \sum_{k=0}^{2} p_{k}(t) \frac{\partial}{\partial w_{i}} f_{k}\left(t, s^{*}(t), w^{*}(t)\right)=\frac{\beta\left(\gamma_{i}^{*}(t)\right)}{\psi\left(\gamma_{i}^{*}(t)\right)}-\frac{p_{i}(t)}{\psi\left(\gamma_{i}^{*}(t)\right) \cdot \cos \theta_{i}(t)} \quad i=1,2,
$$

from the optimality condition (5.10) it follows

$$
\xi_{1}(t)=\xi_{2}(t)=\frac{\mathrm{d}}{\mathrm{d} \varepsilon}\left[\min _{w_{1}+w_{2} \leq 1+\varepsilon} \sum_{i=0}^{2} p_{i}(t) \cdot f_{i}\left(t, s^{*}(t), w\right)\right]_{\varepsilon=0} .
$$

The positive quantity $W(t) \doteq-\xi_{i}(t)$ is the instantaneous value of time.

At the terminal time $t=T^{*}$ one has $s_{1}=s_{2}$ and $\gamma_{1}\left(T^{*}\right)=\gamma_{2}\left(T^{*}\right)=P$. From the necessary condition (4.30), taking into account the orientations of $\mathbf{e}_{1}=-\mathbf{e}$ and $\mathbf{e}_{2}=\mathbf{e}$, it thus follows

$$
\theta_{1}\left(T^{*}\right)=\theta_{2}\left(T^{*}\right) \text {. }
$$

In particular, the control that achieves the minimum in (5.10) is $w_{1}=w_{2}=1 / 2$, hence $f_{0}=\beta / \psi$. To compute the difference $f_{1}-f_{2}$, observe that at the terminal point $P$ one has

$$
f_{1}\left(T^{*}, s_{1}\left(T^{*}\right), \frac{1}{2}\right)-f_{2}\left(T^{*}, s_{2}\left(T^{*}\right), \frac{1}{2}\right)=\frac{\cos \theta_{i}\left(T^{*}\right)}{\psi(P)},
$$

where $\theta_{1}, \theta_{2}$ are the angles between the barriers $\gamma_{1}, \gamma_{2}$ and the fire front, as in Figure 5 . At the terminal time $t=T^{*}$ these two angles are equal, and can be determined by the identity

$$
h(P)=\frac{\sin \theta_{i}\left(T^{*}\right)}{2 \psi(P)} .
$$

Using these relations in (5.12) we obtain

$$
\frac{\beta(P)}{\psi(P)}-\eta \frac{\cos \theta_{i}\left(T^{*}\right)}{\psi(P)}=0, \quad \eta=\frac{\beta(P)}{\cos \theta_{i}\left(T^{*}\right)}
$$




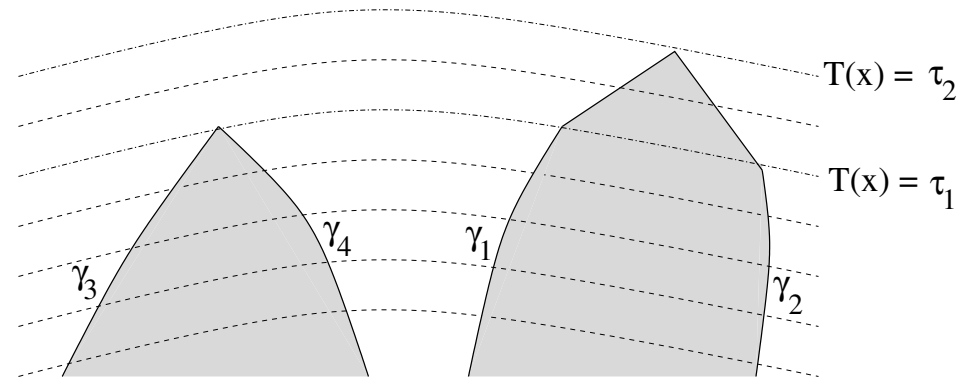

FiguRE 6. Four boundary arcs, joining at different times.

According to (4.30), the terminal value of time is computed by

$$
W\left(T^{*}\right)=\frac{1}{\cos \theta_{i}} \frac{\eta}{\psi}-\frac{\beta}{\psi}=\left(\frac{1}{\cos ^{2} \theta_{i}\left(T^{*}\right)}-1\right) \frac{\beta(P)}{\psi(P)}=\frac{4 \psi^{2}(P) h^{2}(P)}{1-4 \psi^{2}(P) h^{2}(P)} \cdot \frac{\beta(P)}{\psi(P)}>0 .
$$

Indeed, by (5.14) we have $\cos ^{2} \theta_{i}\left(T^{*}\right)=1-4 \psi^{2}(P) h^{2}(P)$.

For future use, we shall denote by $V\left(\tau, \bar{s}_{1}, \bar{s}_{2}\right)$ the value function, i.e. the minimum cost corresponding to initial data

$$
s(\tau)=\bar{s}_{1}, \quad s_{2}(\tau)=\bar{s}_{2} .
$$

\subsection{Four boundary arcs joining at different times}

Next, we study the case of four boundary arcs, shown in Figure 6 . The two arcs $\gamma_{3}, \gamma_{4}$ join together at a time $\tau_{1}$, while $\gamma_{1}$ and $\gamma_{2}$ join at a later time $\tau_{2}>\tau_{1}$.

This can be modelled by a control system with state $s=\left(s_{0}, s_{1}, s_{2}, s_{3}, s_{4}\right)$ and control functions $w=$ $\left(w_{1}, w_{2}, w_{3}, w_{4}\right)$.

$$
\text { Minimize: } s_{0}(T)+V\left(T, s_{1}(T), s_{2}(T)\right)
$$

for the system with dynamics

$$
\left\{\begin{array}{rl}
\dot{s}_{0} & =f_{0}(t, s, w) \\
\dot{s}_{i} & =f_{i}\left(t, s_{i}, w_{i}\right)
\end{array} \quad i=1,2,3,4\right.
$$

Here $V\left(\tau, \bar{s}_{1}, \bar{s}_{2}\right)$ is the value function corresponding to the previous problem with two walls, considered at (5.15). Moreover,

$$
f_{0}(t, s, w)=\sum_{i=1}^{4} \beta\left(x\left(t, s_{i}(t)\right)\right) \frac{w_{i}(t)}{\psi\left(x\left(t, s_{i}(t)\right)\right)}+\left(\int_{s_{1}(t)}^{s_{2}(t)}+\int_{s_{3}(t)}^{s_{4}(t)}\right) h(x(t, \xi)) \alpha(x(t, \xi)) \mathrm{d} \xi,
$$

while the functions $f_{1}, \ldots, f_{4}$ are implicitly determined by the identities

$$
\left|\frac{\partial x\left(t, s_{i}\right)}{\partial t}+f_{i}\left(t, s_{i}, w_{i}\right) \cdot \frac{\partial x\left(t, s_{i}\right)}{\partial s_{i}}\right|=\frac{w_{i}}{\psi\left(x\left(t, s_{i}\right)\right)} \quad i=1,2,3,4 .
$$

The controls satisfy the constraints

$$
w_{i}(t) \in[0,1], \quad \sum_{i=1}^{4} w_{i}(t) \leq 1
$$


while the terminal set is described by the identity:

$$
s_{3}(T)-s_{4}(T)=0 .
$$

Let $t \mapsto w^{*}(t)$ be an optimal control, with optimal trajectory $t \mapsto s^{*}(t)$. Then by the Pontryagin necessary conditions $[7,12,14]$, there exists a nontrivial absolutely continuous adjoint vector $p(t) \doteq\left(p_{0}, p_{1}, p_{2}, p_{3}, p_{4}\right)(t)$ such that

$$
\begin{gathered}
\dot{p}_{0}(t)=0 \quad \dot{q}_{i}(t)=-\sum_{k=0}^{4} p_{k} \frac{\partial f_{k}}{\partial s_{i}}, \quad i=1, \ldots, 4, \\
\sum_{i=0}^{4} p_{i}(t) \cdot f_{i}\left(t, s^{*}(t), w^{*}(t)\right)=\min _{w_{1}+w_{2}+w_{3}+w_{4} \leq 1} \sum_{i=0}^{4} p_{i}(t) \cdot f_{i}\left(t, s^{*}(t), w\right)
\end{gathered}
$$

at almost every time $t$. In addition, at the terminal time $t=\tau_{1}$ one has

$$
\left(p_{0}, p_{1}, p_{2}, p_{3}, p_{4}\right)\left(\tau_{1}\right)=\left(1, V_{s_{1}}, V_{s_{2}},-\eta, \eta\right)
$$

for some real number $\eta$. This Lagrange multiplier can be determined using the further relation

$$
\min _{w_{1}+w_{2}+w_{3}+w_{4} \leq 1} \sum_{i=0}^{4} p_{i}\left(\tau_{1}\right) \cdot f_{i}\left(\tau_{1}, s^{*}\left(\tau_{1}\right), w\right)=0 .
$$

From the minimality condition (5.23) it follows that, by setting

$$
\xi_{j}(t) \doteq \sum_{i=0}^{4} p_{i}(t) \cdot \frac{\partial}{\partial w_{j}} f_{i}\left(t, s^{*}(t), w^{*}(t)\right)=\frac{-p_{j}(t)}{\cos \theta_{j}(t) \psi\left(\gamma_{j}(t)\right)}-\frac{\beta\left(\gamma_{j}(t)\right)}{\psi\left(\gamma_{j}(t)\right)},
$$

one has

$$
\xi_{1}(t)=\xi_{2}(t)=\xi_{3}(t)=\xi_{4}(t)=\frac{\mathrm{d}}{\mathrm{d} \varepsilon}\left[\min _{w_{1}+w_{2}+w_{3}+w_{4} \leq 1+\varepsilon} \sum_{i=0}^{4} p_{i}(t) \cdot f_{i}\left(t, s^{*}(t), w(t)\right)\right]_{\varepsilon=0} .
$$

As in the previous case, the positive quantity $W(t) \doteq-\xi_{i}(t)$ yields the instantaneous value of time.

\subsection{Junctions between a free arc and a boundary arc}

We now consider a free $\operatorname{arc} \gamma$ and a boundary $\operatorname{arc} \gamma^{\sharp}$, joining at a point $P$, as shown in Figure 7. In [4] it was proved that, if these arcs are part of an optimal strategy minimizing the total burned area, then they must be tangent at $P$. Here we study a more general case and derive further necessary conditions for optimality.

To fix the ideas, we assume that $T^{\Gamma}(x) \leq \tau_{1}$ for all $x \in \gamma$ and $T^{\Gamma}(x) \geq \tau_{1}$ for all $x \in \gamma^{\sharp}$. We assume that the free arc $\gamma$ is normal and parameterized by arc-length: $s \mapsto \gamma(s)$ with $s \in[a, b]$. It joins the boundary $\operatorname{arc} \gamma^{\sharp}$ at the endpoint $P=\gamma(b)$. As in Figure 2, we denote by $\mathbf{t}(s)$ and by $\mathbf{n}(s)$ respectively the unit tangent vector and the unit normal vector to $\gamma$ and the at the point $\gamma(s)$. As in (3.1), let $\varphi^{\sharp}:[a, b] \mapsto \mathbb{R}$ be a smooth function which vanishes in a neighborhood of $a$ and $b$, and such that (3.2) holds.

Given any vector $\mathbf{v} \in \mathbb{R}^{2}$ and scalar $\rho \in \mathbb{R}$, we shall construct a family of perturbed curves $\gamma_{\varepsilon}:[a, b] \mapsto \mathbb{R}^{2}$ having endpoints

$$
\gamma_{\varepsilon}(a), \quad \gamma_{\varepsilon}(b)+\varepsilon \mathbf{v},
$$

and such that the total time needed to construct each $\gamma_{\varepsilon}$ is

$$
\mathcal{T}\left(\gamma_{\varepsilon}\right)=\mathcal{T}(\gamma)+\varepsilon \rho+o(\varepsilon) .
$$




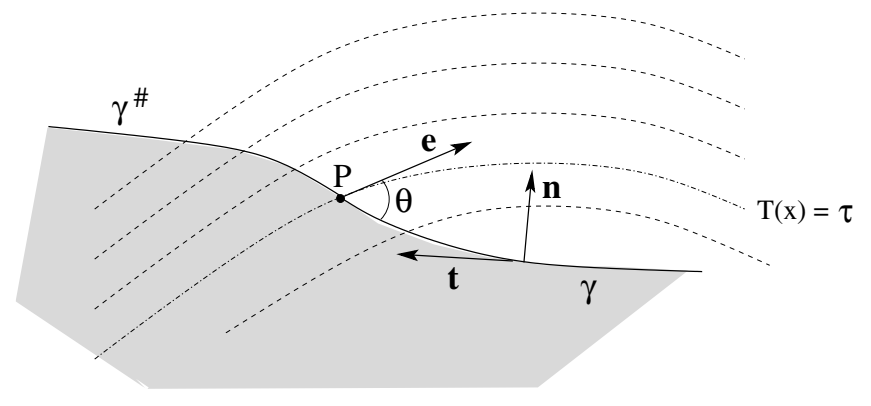

FIGURE 7. A junction between a free $\operatorname{arc} \gamma$ and a boundary $\operatorname{arc} \gamma^{\sharp}$.

Here and in the sequel, the Landau symbol $o(\varepsilon)$ denotes an infinitesimal of higher order w.r.t. $\varepsilon$. Toward this goal, choose a smooth function $\varphi:[a, b] \mapsto \mathbb{R}^{2}$ such that

$$
\varphi(b)=\mathbf{v}, \quad \varphi(s)=0 \text { for } s \text { in a neighborhood of } a,
$$

and let $\varphi_{1}, \varphi_{2}$ be the normal and the tangential components of $\varphi$, so that

$$
\varphi(s)=\varphi_{1}(s) \mathbf{n}(s)+\varphi_{2}(s) \mathbf{t}(s) .
$$

Moreover, define

$$
\begin{gathered}
\eta_{0}^{\prime} \doteq\left(\int_{a}^{b} \mathcal{G}(\psi, \gamma, s) \varphi^{\sharp}(s) \mathrm{d} s\right)^{-1}\left(\rho-\psi(\gamma(b)) \varphi_{2}(b)-\int_{a}^{b} \mathcal{G}(\psi, \gamma, s) \varphi_{1}(s) \mathrm{d} s\right), \\
\gamma_{\varepsilon}(s) \doteq \gamma(s)+\varepsilon \varphi(s)+\varepsilon \eta_{0}^{\prime} \varphi^{\sharp}(s) \mathbf{n}(s) \quad
\end{gathered}
$$

Recalling that $\varphi^{\sharp}$ vanishes at $s=a$ and at $s=b$, it is clear that the end-point conditions (5.28) are satisfied. We claim that (5.29) also holds.

Since now we are perturbing $\gamma$ also in the tangential direction, computing the derivative of the time needed to construct $\gamma_{\varepsilon}$ as in (3.5) we obtain an additional term. Namely, calling $\kappa(s)$ the curvature of $\gamma$, in place of (3.5) one has

$$
\begin{aligned}
{\left[\frac{\mathrm{d}}{\mathrm{d} t} \mathcal{T}\left(\gamma_{\varepsilon}\right)\right]_{\varepsilon=0}=} & \int_{a}^{b}\{\langle\nabla \psi(\gamma(s)), \mathbf{n}(s)\rangle+\psi(\gamma(s)) \kappa(\gamma(s))\} \cdot\left(\varphi_{1}(s)+\eta_{0}^{\prime} \varphi^{\sharp}(s)\right) \mathrm{d} s \\
& +\int_{a}^{b}\left\{\langle\nabla \psi(\gamma(s)), \mathbf{t}(s)\rangle \varphi_{2}(s)+\psi(\gamma(s))\left\langle\mathbf{t}(s), \frac{\mathrm{d}}{\mathrm{d} s}\left[\varphi_{2}(s) \mathbf{t}(s)\right]\right\rangle\right\} \mathrm{d} s=I_{1}+I_{2} .
\end{aligned}
$$

We observe that the identity (3.17) was true for every scalar function $\phi$ vanishing at the endpoints $a, b$. By a density argument, it still holds for a smooth function $\varphi_{1}$ which does not vanish at $s=b$. Using this identity, together with $\langle\mathbf{t}(s), \dot{\mathbf{t}}(s)\rangle \equiv 0$, we obtain

$$
\begin{aligned}
& I_{1}=\int_{a}^{b} \mathcal{G}(\psi, \gamma, s)\left(\varphi_{1}(s)+\eta_{o}^{\prime} \varphi^{\sharp}(s)\right) \mathrm{d} s=\rho-\psi(\gamma(b)) \varphi_{2}(b), \\
& I_{2}=\int_{a}^{b}\left\{\frac{\mathrm{d}}{\mathrm{d} s}\left(\psi(\gamma(s)) \varphi_{1}(s)\right)\right\} \mathrm{d} s=\psi(\gamma(b)) \varphi_{2}(b) .
\end{aligned}
$$

In connection with (5.32), this yields (5.29). 
We now consider the cost $J\left(\gamma_{\varepsilon}\right)$ associated with the perturbed arc $\gamma_{\varepsilon}$. The change in the cost of building the wall is described by

$$
\begin{aligned}
\frac{\mathrm{d}}{\mathrm{d} \varepsilon}\left[\int_{a}^{b} \beta\left(\gamma_{\varepsilon}(s)\right)\left|\dot{\gamma}_{\varepsilon}(s)\right| \mathrm{d} s\right]_{\varepsilon=0}= & \int_{a}^{b}\{\langle\nabla \beta(\gamma(s)), \mathbf{n}(s)\rangle+\beta(\gamma(s)) \kappa(\gamma(s))\} \cdot\left(\varphi_{1}(s)+\eta_{0}^{\prime} \varphi^{\sharp}(s)\right) \mathrm{d} s \\
& +\int_{a}^{b}\left\{\frac{\mathrm{d}}{\mathrm{d} s}\left[\beta(\gamma(s)) \varphi_{2}(s)\right]\right\} \mathrm{d} s .
\end{aligned}
$$

On the other hand, the change in the cost related to the burned area is estimated by

$$
\varepsilon \int_{a}^{b} \alpha(\gamma(s))\left(\varphi_{1}(s)+\eta_{0}^{\prime} \varphi^{\sharp}(s)\right) \mathrm{d} s+o(\varepsilon) .
$$

We now use the fact that the free $\operatorname{arc} \gamma$ is optimal, hence (3.12) holds (with $\varphi$ replaced by $\varphi_{1}$ ). Comparing (5.35)(5.36) with (5.30), and using (3.19), we obtain

$$
\left.\frac{\mathrm{d}}{\mathrm{d} \varepsilon} J\left(\gamma_{\varepsilon}\right)\right|_{\varepsilon=0}=\varphi_{2}(b)(\psi \cdot \eta+\beta)-\rho \eta,
$$

where $\eta$ is the Lagrange multiplier defined at (3.13). It is understood that the functions $\psi, \beta$ are here computed at the terminal point $\gamma(b)$.

Remark 5.1. The value $\lambda$ in (3.13) and (3.19) describes the value of time, which remains constant as long as the free arc is being built. The formula (5.37) thus has a simple interpretation. If the free arc $\gamma$ is part of an optimal strategy, the only change in the cost functional associated to the perturbation $\gamma_{\varepsilon}$ is due to (i) the cost of building the additional portion of wall near the endpoint $\gamma(b)$, and (ii) the change in the construction time.

We can now state the main result of this section.

Theorem 5.1 (necessary conditions at junctions). Let $\gamma_{1}, \ldots, \gamma_{\nu} \subset \Gamma_{\mathcal{F}} \backslash \Gamma^{d}$ be free arcs, simultaneously constructed by an optimal strategy $\Gamma$ during the time interval $t \in] \tau_{0}, \tau_{1}[$. Assume that at least one of these arcs is normal, and let $s \mapsto \gamma_{i}(s)$ be a parameterization of $\gamma_{i}$ by arc-length, with $\left.s \in\right] a_{i}, b_{i}[$.

Let $\gamma_{1}^{*}, \ldots, \gamma_{\nu}^{*} \subset \Gamma_{S} \backslash \Gamma^{d}$ be boundary arcs, all normal, simultaneously constructed by the optimal strategy during the time interval $t \in\left[\tau_{1}, \tau_{2}\right]$.

Assume that each pair of arcs $\gamma_{i}, \gamma_{i}^{*}$ have a common endpoint

$$
P_{i}=\gamma_{i}\left(b_{i}\right)=\gamma_{i}^{*}\left(\tau_{1}\right)
$$

and that the angle $\theta_{i}$ between the barrier $\gamma_{i}$ and the fire front $\left\{T^{\Gamma}(x)=\tau_{1}\right\}$ at the junction point $P_{i}$ (see Fig. 7 ) satisfies

$$
0<\theta_{i}<\frac{\pi}{2} \quad i=1, \ldots, \nu .
$$

Then there exists constants $\lambda_{0}=1$ and $\lambda$, and adjoint variables $q_{i}(t)$, such that all necessary conditions stated in Theorems 3.1 and 4.1 hold, together with the following matching conditions, valid for $i=1, \ldots, \nu$.

$$
\begin{gathered}
q_{i}\left(\tau_{1}\right)=\left(\beta\left(P_{i}\right)+\lambda \psi\left(P_{i}\right)\right) \cos \theta_{i}, \\
W_{i}\left(\tau_{1}\right)=\lambda
\end{gathered}
$$

Moreover, for each $i$ the curves $\gamma_{i}$ and $\gamma_{i}^{*}$ meet tangentially at the point $P_{i}$. 

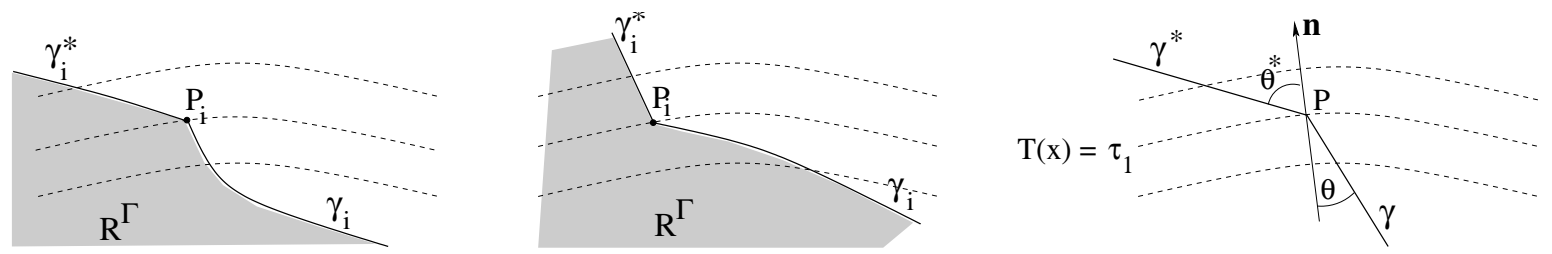

Figure 8. Left: the two $\operatorname{arcs} \gamma_{i}, \gamma_{i}^{\sharp}$ produce an outward corner. Center: two arcs producing an inward corner. Right: the angles $\theta, \theta^{*}$ formed by the $\operatorname{arcs} \gamma, \gamma^{\sharp}$ with the normal vector $\mathbf{n}$ to the level set $T(x)=\tau_{1}$ at $P$.

Remark 5.2. We recall that $W_{i}(t)$ is the instantaneous value of time for the boundary arc $\gamma_{i}^{*}$, defined at (4.30). This function is the same for all $i=1, \ldots, \nu$, but can decrease in time. On the other hand, $\lambda$ is the constant value of time corresponding to the free arcs. The identity (5.41) says that the value of time is continuous at $t=\tau_{1}$, when the junction occurs.

To motivate the identities (5.40), we observe that the Lagrange multiplier $q_{i}(t)$ describes the increase in the total cost produced by shifting the initial point of the boundary arc $\gamma_{i}^{*}(t)$ in the direction of the unit vector $\mathbf{e}_{i}\left(\tau_{1}\right)$. On the other hand (5.37) implies that, if we shift the terminal point of the free arc $\gamma_{i}$ in the direction $\mathbf{e}_{i}\left(\tau_{1}\right)=v_{1} \mathbf{n}-v_{2} \mathbf{t}$ without changing the total construction time (i.e. with $\rho=0$ ), the cost related to the free arc decreases at the rate

$$
v_{2} \beta\left(\gamma_{i}\left(b_{i}\right)\right)+\lambda v_{2} \psi\left(\gamma_{i}\left(b_{i}\right)\right)=\left(\beta\left(P_{i}\right)+\eta \psi\left(P_{i}\right)\right) \cos \theta_{i}
$$

Proof.

1. We begin by showing that, for every $i=1, \ldots, \nu$, the $\operatorname{arcs} \gamma_{i}$ and $\gamma_{i}^{*}$ are tangent at the junction point $P_{i}$.

If not, we claim that there exists at least one index $i$ such that at $P_{i}$ the $\operatorname{arcs} \gamma_{i}, \gamma_{i}^{*}$ produce an inward corner (see Fig. 8).

Indeed, consider the portion of the arc $\gamma_{i}$ which is reached by the fire during the interval $[\tau-\varepsilon, \tau]$. This has length

$$
m_{1}\left(\left\{x \in \gamma_{i} ; \quad T(x) \in\left[\tau_{1}-\varepsilon, \tau_{1}\right]\right\}\right)=\frac{\varepsilon h\left(P_{i}\right)}{\cos \theta_{i}}+o(\varepsilon),
$$

where $h\left(P_{i}\right)$ is the speed at which the fire front is advancing, at the point $P_{i}$, as in (2.6). Similarly,

$$
m_{1}\left(\left\{x \in \gamma_{i}^{*} ; \quad T(x) \in\left[\tau_{1}, \tau_{1}+\varepsilon\right]\right\}\right)=\frac{\varepsilon h\left(P_{i}\right)}{\cos \theta_{i}^{*}}+o(\varepsilon)
$$

By assumption, the constraint (1.10) is satisfied as an equality for $t \geq \tau_{1}$ and as a strict inequality for $t<\tau_{1}$. For $\varepsilon>0$ small, this yields

$$
\begin{aligned}
& \varepsilon=\int_{\Gamma \cap\left\{T(x) \in\left[\tau_{1}, \tau_{1}+\varepsilon\right]\right\}} \psi \mathrm{d} m_{1}=\sum_{i=1}^{\nu} \psi\left(P_{i}\right) \frac{\varepsilon h\left(P_{i}\right)}{\cos \theta_{i}^{*}}+o(\varepsilon), \\
& \varepsilon<\int_{\Gamma \cap\left\{T(x) \in\left[\tau_{1}-\varepsilon, \tau_{1}\right]\right\}} \psi \mathrm{d} m_{1}=\sum_{i=1}^{\nu} \psi\left(P_{i}\right) \frac{\varepsilon h\left(P_{i}\right)}{\cos \theta_{i}}+o(\varepsilon) .
\end{aligned}
$$

If now $0 \leq \theta_{i} \leq \theta_{i}^{*} \leq \pi / 2$ for every $i=1, \ldots, \nu$, and $\theta_{j}<\theta_{j}^{*}$ for at least one $j$, then the two above conditions yield a contradiction. 

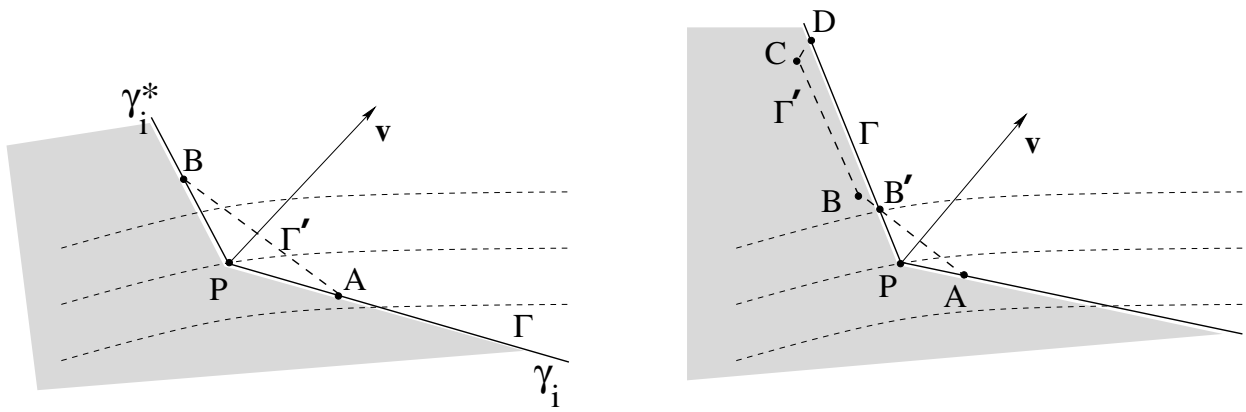

Figure 9. If a barrier $\Gamma$ contains an inward corner, it can be replaced by a barrier $\Gamma^{\prime}$ yielding a smaller cost.

2. By the previous step, we can now assume that at least one pair of arcs, say $\gamma_{i}$ and $\gamma_{i}^{*}$, form an inward corner. We claim that this blocking strategy $\Gamma$ is not optimal.

Indeed, if the construction cost is strictly positive, i.e. $\beta\left(P_{i}\right)>0$, then a strictly better strategy $\Gamma^{\prime}$ is as follows (Fig. 9, left). Fix $\varepsilon>0$ small. Let $A$ be the point along $\gamma_{i}$ at distance $\varepsilon$ from $P_{i}$. Let $B$ be the point along $\gamma_{i}^{*}$ such that the segment $A B$ is perpendicular to the bisectrix $\mathbf{v}$ of the angle at $P_{i}$. Replace the portion of walls in $\gamma_{i} \cup \gamma_{i}^{*}$ between $A$ and $B$ by this segment. The new barrier $\Gamma^{\prime}$ thus obtained is admissible. The length of $\Gamma^{\prime}$ satisfies $m_{1}\left(\Gamma^{\prime}\right) \leq m_{1}(\Gamma)-c \varepsilon$, for some $c>0$ an all $\varepsilon>0$ small enough. On the other hand, the additional area burned by the fire is of order $\mathcal{O}\left(\varepsilon^{2}\right)$. Hence, if $\beta\left(P_{i}\right)>0$, for $\varepsilon>0$ small the total cost associated with $\Gamma^{\prime}$ is $J\left(\Gamma^{\prime}\right)<J(\Gamma)$.

On the other hand, if $\beta\left(P_{i}\right)=0$ but $\alpha\left(P_{i}\right)>0$, then we can reduce both the total length of the wall and the total area burned by the fire, as shown in Figure 9 , right. Fix $\varepsilon>0$ small. Let $A$ be the point along $\gamma_{i}$ at distance $\varepsilon$ from $P_{i}$. Construct a segment $A B^{\prime}$ perpendicular to the bisectrix v. Prolong this segment to a point $B$ such that (with obvious meaning of notation) the length of the various arcs satisfy

$$
m_{1}(A B)+2 m_{1}\left(B^{\prime} B\right)=m_{1}\left(A P_{i}\right)+m_{1}\left(P_{i} B^{\prime}\right)
$$

Then construct an arc $B^{\prime} C$ of length $\kappa \varepsilon$ (with $\kappa \gg 1$ ), having constant distance to $\gamma_{i}^{*}$. Finally, connect the point $C$ with a point $D$ on $\gamma_{i}^{*}$.

The new barrier $\Gamma^{\prime}$ obtained by replacing the $\operatorname{arcs} A P_{i}$ and $P_{i} D$ with $A B \cup B C \cup C D$ is still admissible. Its total length is smaller, and the total burned area has also decreased. Indeed, by choosing $\kappa$ large enough, the area of the region $B^{\prime} B C D$ is strictly smaller than the area of the triangle $A P_{i} B^{\prime}$. Hence, for $\varepsilon>0$, we again conclude that $J\left(\Gamma^{\prime}\right)<J(\Gamma)$, against the optimality of the strategy $\Gamma$. The above arguments prove the last statement of the theorem: for every $i=1, \ldots, \nu$, the $\operatorname{arcs} \gamma_{i}$ and $\gamma_{i}^{*}$ are tangent at the point of junction.

3. Toward a proof of the matching conditions (5.40), we remark that the optimality conditions for boundary arcs in Theorem 4.1 were obtained by considering an auxiliary optimal control problem with fixed endpoints. However, the analysis in (5.28)-(5.37) shows that, for any choice of the numbers $r_{1}, \ldots, r_{\nu}$, we can replace the free $\operatorname{arcs} \gamma_{i}$ by perturbed $\operatorname{arcs} \gamma_{i, \varepsilon}$, terminating at the endpoints $x_{i}\left(\tau_{1}, \varepsilon r_{i}\right)$. Here we use the coordinates $(t, s) \mapsto x_{i}(t, s)$ as in Figure 3. More precisely, the following holds.

(i) The arc $\gamma_{i, \varepsilon}$ starts at $\gamma_{i}\left(a_{i}\right)$ and terminates at the point

$$
\gamma_{i, \varepsilon}\left(b_{i}\right)=x_{i}\left(\tau_{1}, \varepsilon r_{i}\right)=P_{i}+\varepsilon r_{i} \mathbf{e}_{i}\left(\tau_{1}\right)+o(\varepsilon)
$$


(ii) The total amount of time needed to construct these arcs is the same:

$$
\sum_{i=1}^{\nu} \mathcal{T}\left(\gamma_{i, \varepsilon}\right) \doteq \sum_{i=1}^{\nu} \int_{a_{i}}^{b_{i}} \psi_{i}\left(\gamma_{i, \varepsilon}(s)\right) \mathrm{d} s=\sum_{i=1}^{\nu} \int_{a_{i}}^{b_{i}} \psi_{i}\left(\gamma_{i}(s)\right) \mathrm{d} s \doteq \sum_{i=1}^{\nu} \mathcal{T}\left(\gamma_{i}\right)
$$

(iii) As in (5.37), the change in cost associated with these perturbed arcs is

$$
\left.\frac{\mathrm{d}}{\mathrm{d} \varepsilon} J\left(\gamma_{i, \varepsilon}\right)\right|_{\varepsilon=0}=\left(\beta\left(P_{i}\right)+\lambda \psi\left(P_{i}\right)\right) r_{i} \cos \theta_{i}
$$

At this point, the conditions (5.40) become clear. For an optimal control problem with free initial point, the initial values of the adjoint variables $q_{1}, \ldots, q_{\nu}$ should equal the gradients of the cost associated with these initial values.

To make this argument completely rigorous, however, we must clarify a technical point. Indeed, in principle the perturbed strategies consisting of free $\operatorname{arcs} \gamma_{i, \varepsilon}$, then of boundary $\operatorname{arcs} \gamma_{i, \varepsilon}^{\sharp}$ starting at the points $\gamma_{i, \varepsilon}\left(b_{i}\right)=x_{i}\left(\tau_{1}, \varepsilon \alpha_{i}\right)$, may not be admissible. Indeed, since now we are perturbing the free $\operatorname{arcs} \gamma_{i}$ also at points where the constraint (1.10) is saturated, the arguments used at (3.8) and (3.9) now break down.

To take care of this difficulty, for any fixed $\delta>0$ we replace the $\operatorname{arcs} \gamma_{i, \varepsilon}$ with free $\operatorname{arcs} \tilde{\gamma}_{i, \varepsilon}$ having the same endpoints

$$
\tilde{\gamma}_{i, \varepsilon}\left(a_{i}\right)=\gamma_{i, \varepsilon}\left(a_{i}\right), \quad \tilde{\gamma}_{i, \varepsilon}\left(b_{i}\right)=\gamma_{i, \varepsilon}\left(b_{i}\right),
$$

but requiring slightly shorter time to construct. Namely

$$
\sum_{i=1}^{\nu} \mathcal{T}\left(\tilde{\gamma}_{i, \varepsilon}\right)=\sum_{i=1}^{\nu} \mathcal{T}\left(\gamma_{i}\right)-\delta|\varepsilon| .
$$

According to (5.37), the cost associated with these new free arcs is

$$
\sum_{i=1}^{\nu} J\left(\tilde{\gamma}_{i, \varepsilon}\right)=\sum_{i=1}^{\nu} J\left(\gamma_{i}\right)+\varepsilon \sum_{i=1}^{\nu} r_{i}\left(\beta\left(P_{i}\right)+\lambda \psi\left(P_{i}\right)\right) \cos \theta_{i}+\lambda \delta|\varepsilon| .
$$

Because of (5.44), for any fixed $\delta>0$ these alternative strategies will be admissible, for $\varepsilon$ in a neighborhood of the origin (possibly shrinking to zero as $\delta \rightarrow 0$ ).

For $\left(s_{1}, \ldots, s_{\nu}\right) \approx(0, \ldots, 0)$, we now define

$$
V\left(s_{1}, \ldots, s_{\nu}\right) \doteq \inf \sum_{i=1}^{\nu} J\left(\tilde{\gamma}_{i}\right)
$$

where the infimum is taken over all admissible $\nu$-tuples of free $\operatorname{arcs} \tilde{\gamma}_{1}, \ldots, \tilde{\gamma}_{\nu}$, with

$$
\tilde{\gamma}_{i}\left(a_{i}\right)=\gamma_{i}\left(a_{i}\right), \quad \tilde{\gamma}_{i}\left(b_{i}\right)=x_{i}\left(\tau_{1}, s_{i}\right) .
$$

Since $\delta>0$ was arbitrary, the previous argument shows that

$$
\left.\frac{\partial V}{\partial s_{i}}\right|_{\left(s_{1}, \ldots, s_{\nu}\right)=(0, \ldots, 0)}=\left(\beta\left(P_{i}\right)+\lambda \psi\left(P_{i}\right)\right) \cos \theta_{i} .
$$

Since this partial derivative must coincide with the initial value of the adjoint variable $q_{i}$, the identities in (5.40) hold. 
4. Finally, by (4.30) and (5.43) one finds

$$
W_{i}\left(\tau_{1}\right)=\frac{1}{\cos \theta_{i}} \frac{q_{i}}{\psi}-\frac{\beta}{\psi}=\frac{1}{\cos \theta_{i}} \frac{(\beta+\lambda \psi) \cos \theta_{i}}{\psi}-\frac{\beta}{\psi}=\lambda,
$$

proving the matching condition (5.41).

\section{EXAMPLES}

This final section provides three examples, where the value of time and the matching conditions can be directly computed.

Example 6.1. Assume that $F(x) \equiv B(0,1)$, so that the fire propagates with unit speed in all directions.

Assume that, without barriers, the minimal time function is $T(x)=x_{2}$. We take here $\alpha(x) \equiv 1$ and $\beta(x) \equiv 0$, so that we simply seek to minimize the total burned area. Moreover, we assume that the construction speed $\sigma=1 / \psi$ is a constant. We consider two cases.

Case 1. At time $t$, the boundary of the burned region where fire is advancing is a single segment:

$$
\left\{\left(x_{1}, x_{2}\right) ; \quad x_{1} \in\left[s_{1}(t), s_{2}(t)\right], \quad x_{2}=t\right\} .
$$

In this case, assuming that $\sigma>2$, the optimal strategy is to construct the two walls at same speed $\sigma / 2$. If at time $t=0$ we have $\left[s_{1}(0), s_{2}(0)\right]=\left[\bar{s}_{1}, \bar{s}_{2}\right]$, then the time needed to block the fire is

$$
T=\frac{\bar{s}_{2}-\bar{s}_{1}}{2 \sqrt{(\sigma / 2)^{2}-1}}=\frac{\bar{s}_{2}-\bar{s}_{1}}{\sqrt{\sigma^{2}-4}}
$$

The total burned area is

$$
V\left(\bar{s}_{2}-\bar{s}_{1}\right)=\frac{\left(\bar{s}_{2}-\bar{s}_{1}\right)^{2}}{2 \sqrt{\sigma^{2}-4}}
$$

The adjoint variables are

$$
q_{2}(t)=\frac{\partial V}{\partial \bar{s}_{2}}=\frac{s_{2}(t)-s_{1}(t)}{\sqrt{\sigma^{2}-4}}=-\frac{\partial V}{\partial \bar{s}_{1}}=-q_{1}(t)
$$

The angle $\theta$ between each wall and the fire front is determined by

$$
\sin \theta=\frac{2}{\sigma}, \quad \cos \theta=\frac{\sqrt{\sigma^{2}-4}}{\sigma} .
$$

The instantaneous value of time is computed as

$$
W(t)=\frac{q_{2}(t)}{\psi \cos \theta}=\frac{\sigma^{2}}{\sigma^{2}-4} \cdot\left(s_{2}(t)-s_{1}(t)\right) .
$$

Case 2. Assume that at a given time $t$, the boundary of the burned set consists of two segments:

$$
\partial R(t)=\left\{\left(x_{1}, x_{2}\right) ; \quad x_{1} \in\left[s_{1}(t), s_{2}(t)\right] \cup\left[s_{3}(t), s_{4}(t)\right], \quad x_{2}=t\right\} .
$$

We are thus constructing four walls, at the points $P_{i}(t)=\left(s_{i}(t), t\right)$. Assume that $\sigma>4$ and, to fix the ideas, let $s_{4}-s_{3} \leq s_{2}-s_{1}$ (see Fig. 10). We shall reformulate the above problem as an optimal control problem with free terminal time (the time where the walls at $P_{3}$ and $P_{4}$ join together). 


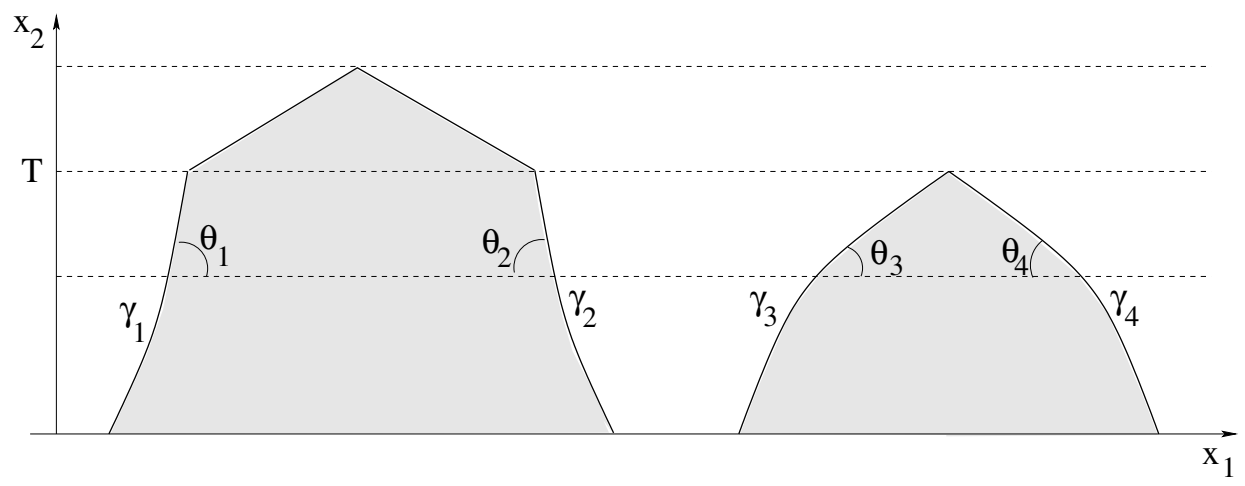

Figure 10. At time $t=T$ the two walls $\gamma_{3}, \gamma_{4}$ join together. The angles $\theta_{1}=\theta_{2}$ increase as $t \rightarrow T$-, while $\theta_{3}=\theta_{4}$ decrease. For $t>T$, all resources are allocated to the construction of the remaining barriers $\gamma_{1}, \gamma_{2}$. In this case, $\theta_{1}(t)=\theta_{2}(t)=\arcsin (2 / \sigma)$.

We set $y_{1}=s_{2}-s_{1}, y_{2}=s_{4}-s_{3}$, while $y_{0}$ will keep track of the burned area up to time $t$. It is clear that an optimal strategy will satisfy $\dot{s}_{2}=-\dot{s}_{1}, \dot{s}_{4}=-\dot{s}_{3}$. Therefore, the above variables evolve in time according to

$$
\left\{\begin{array}{l}
\dot{y}_{0}=y_{1}+y_{2} \\
\dot{y}_{1}=-\sqrt{u_{1}^{2}-4} \\
\dot{y}_{2}=-\sqrt{u_{2}^{2}-4}
\end{array} \quad u_{i} \in[2, \sigma], \quad u_{1}+u_{2} \leq \sigma,\right.
$$

with initial data

$$
y_{1}(0)=\bar{s}_{2}-\bar{s}_{1}, \quad y_{2}(0)=\bar{s}_{4}-\bar{s}_{3}, \quad y_{0}(0)=0 .
$$

The terminal set is

$$
S=\left\{\left(y_{0}, y_{1}, y_{2}\right) ; \quad \phi_{1}\left(y_{0}, y_{1}, y_{2}\right) \doteq y_{2}=0\right\}
$$

and the terminal payoff is

$$
\phi_{0}\left(y_{0}, y_{1}, y_{2}\right)=y_{0}+\frac{y_{1}^{2}}{2 \sqrt{\sigma^{2}-4}} .
$$

Indeed, after the time where the first couple of walls join together, the problem is reduced to optimizing the construction of the two remaining barriers. For this problem, discussed in Case 1, the corresponding optimal value function was computed at (6.1).

Applying the Pontryagin maximum principle to the optimal control problem (6.3)-(6.6), we obtain an adjoint vector $p=\left(p_{0}, p_{1}, p_{2}\right)$ such that

$$
\left\{\begin{array}{l}
\dot{p}_{0}=0 \\
\dot{p}_{1}=-p_{0} \\
\dot{p}_{2}=-p_{0}
\end{array}\right.
$$

with terminal conditions

$$
\left(p_{0}, p_{1}, p_{2}\right)(T)=\nabla \phi_{0}+\lambda_{1} \nabla \phi_{1}=\left(1, \frac{y_{1}}{\sqrt{\sigma^{2}-4}}, \lambda_{1}\right)
$$

for some constant $\lambda_{1}$. Moreover, at every time $t$ one has

$$
\min _{u_{1}, u_{2} \in[2, \sigma], u_{1}+u_{2} \leq \sigma}\left(p_{0}\left(y_{1}+y_{2}\right)-p_{1} \sqrt{u_{1}^{2}-4}-p_{2} \sqrt{u_{2}^{2}-4}\right)=0 .
$$


Since $p_{0}(t) \equiv 1$, at the terminal time $T$ this yields

$$
\min _{\omega_{1}, \omega_{2} \in[2, \sigma], \omega_{1}+\omega_{2} \leq \sigma}\left(y_{1}-\frac{\sqrt{\omega_{1}^{2}-4}}{\sqrt{\sigma^{2}-4}} y_{1}-\lambda_{1} \sqrt{\omega_{2}^{2}-4}\right)=0 .
$$

The three variables $\omega_{1}=u_{1}(T), \omega_{2}=u_{2}(T)$, and $\lambda_{1}$ can be determined from the equations

$$
\left\{\begin{aligned}
\frac{\omega_{1}}{\sqrt{\omega_{1}^{2}-4} \sqrt{\sigma^{2}-4}} y_{1} & =\frac{\lambda_{1} \omega_{2}}{\sqrt{\omega_{2}^{2}-4}} \\
\omega_{1}+\omega_{2} & =\sigma \\
y_{1}-\frac{\sqrt{\omega_{1}^{2}-4}}{\sqrt{\sigma^{2}-4}} y_{1}-\lambda_{1} \sqrt{\omega_{2}^{2}-4} & =0
\end{aligned}\right.
$$

Observe that, if $y_{1}(\tau)=y_{2}(\tau)$ at some time $\tau$, then by symmetry the optimal control is $u_{1}(t)=u_{2}(t)=\sigma / 2$ for all times $t$. In this case $y_{1}(t)=y_{2}(t)$ for all $t$. In particular, both couples of walls terminate at the same time: $y_{1}(T)=y_{2}(T)$. Throughout the following, we shall consider the case where $y_{1}(t)>y_{2}(t)$ for all $t$.

We now study the existence and uniqueness of solutions to the system (6.11). Given $p_{1}, p_{2}$, if $u_{1}, u_{2}$ achieve the minimum in (6.9) then

$$
p_{1} \cdot \frac{u_{1}}{\sqrt{u_{1}^{2}-4}}=p_{2} \cdot \frac{u_{2}}{\sqrt{u_{2}^{2}-4}} .
$$

At the terminal time $t=T$, since $\omega_{2}=\sigma-\omega_{1}$, from (6.12) it follows

$$
\frac{\omega_{1}}{\sqrt{\omega_{1}^{2}-4}} \cdot \frac{\sqrt{\left(\sigma-\omega_{1}\right)^{2}-4}}{\sigma-\omega_{1}}=\frac{p_{2}}{p_{1}}
$$

We observe that the left hand side of (6.13) is a monotonically decreasing function, for $\left.\omega_{1} \in\right] 2, \sigma-2[$. It approaches $+\infty$ as $\omega_{1} \rightarrow 2+$ and it approaches $-\infty$ as $\omega_{1} \rightarrow(\sigma-2)-$. Therefore for a given ratio $p_{2} / p_{1}>0$, the equation (6.13) has exactly one solution $\omega_{1} \in(2, \sigma-2)$.

We now observe that $p_{1}(T)$ is determined by $y_{1}(T)$, but $p_{2}(T)=\lambda_{1}$ still needs to be determined. At the terminal time $T$, the minimum value

$$
\min _{u_{1}+u_{2} \leq \sigma}\left(y_{1}-\frac{y_{1}}{\sqrt{\sigma^{2}-4}} \sqrt{u_{1}^{2}-4}-\lambda_{1} \sqrt{u_{2}^{2}-4}\right)
$$

is a decreasing function of $\lambda_{1}$. Hence there exists unique $\lambda_{1}$ satisfying (6.9) at $t=T$. Together with the previous analysis, this shows that the system (6.11) has a unique solution.

We now claim that $p_{2}(T)>p_{1}(T)$, i.e. $\lambda_{1}>\frac{y_{1}}{\sqrt{\sigma^{2}-4}}$.

On the contrary, assume that $\lambda_{1} \leq \frac{y_{1}}{\sqrt{\sigma^{2}-4}}$. In view of (6.11),

$$
\frac{y_{1}}{\sqrt{\sigma^{2}-4}} \cdot\left\{\sqrt{\sigma^{2}-4}-\sqrt{\omega_{1}^{2}-4}-\sqrt{\left(\sigma-\omega_{1}\right)^{2}-4}\right\} \leq 0 .
$$

Since the right hand side of (6.14) is convex w.r.t. $\omega_{1}$ and attains the minimum

$$
\frac{y_{1}}{\sqrt{\sigma^{2}-4}} \cdot\left\{\sqrt{\sigma^{2}-4}-2 \sqrt{\left(\frac{\sigma}{2}\right)^{2}-4}\right\} \geq 0
$$

at $\omega_{1}=\sigma / 2$, the above leads to a contradiction with (6.14). Hence $p_{2}(T)>p_{1}(T)$. 
In turn, this implies $\omega_{2}>\sigma / 2>\omega_{1}$. Moreover, $p_{1}(t)=p_{1}(T)+T-t$ and $p_{2}(t)=p_{2}(T)+T-t$. Therefore the ratio satisfies $p_{2}(t) / p_{1}(t)>1$ and is increasing in time, achieving a maximum at the terminal time $t=T$. By (6.12), the optimal controls satisfy $u_{2}(t)>u_{1}(t)$, with $t \mapsto u_{2}(t)$ increasing and $t \mapsto u_{1}(t)$ decreasing, up to time $T$.

Finally, consider the value of time

$$
W(t)=\frac{\sigma q_{i}(t)}{\cos \theta_{i}(t)},
$$

where $\theta_{i}(t)$ is the angle formed by the wall $\gamma_{i}$ and the fire front. Notice that, for $i=1,2$, this can be well defined also for $t>T$. The adjoint variables $q_{1}(t), q_{2}(t)$ remain continuous at the time $t=T$, while the angles $\theta_{1}, \theta_{2}$ suddenly decrease at the time $T$ where the walls $\gamma_{3}$ and $\gamma_{4}$ meet. Therefore, one expects that $W(\cdot)$ should have a downward jump at $t=T$. This is confirmed by the following computations:

$$
q_{1}(T)=q_{2}(T)=\frac{y_{1}(T)}{\sqrt{\sigma^{2}-4}}=\frac{s_{2}(T)-s_{1}(T)}{\sqrt{\sigma^{2}-4}} .
$$

For $t>T$ we have

$$
\theta_{1}(t)=\theta_{2}(t)=\arcsin \frac{2}{\sigma}
$$

hence the value of time is provided by (6.2). In particular

$$
\lim _{t \rightarrow T+} W(t)=\frac{\sigma^{2}}{\sigma^{2}-4}\left(s_{2}(T)-s_{1}(T)\right) .
$$

On the other hand, for $t<T$ we have $p_{2}(t) \geq p_{1}(t)$, hence $u_{2}(t) \geq u_{1}(t)$ and

$$
w_{1}(t)=w_{2}(t)=\frac{u_{1}(t)}{2} \leq \frac{\sigma}{4} .
$$

Hence the angles $\theta_{1}(t)=\theta_{2}(t)$ between the walls $\gamma_{1}, \gamma_{2}$ and the fire front satisfy

$$
\sin \theta_{i}(t) \geq \frac{4}{\sigma}, \quad \frac{1}{\cos \theta_{i}(t)} \geq \frac{\sigma}{\sqrt{\sigma^{2}-16}} .
$$

Therefore

$$
\lim _{t \rightarrow T-} W(t)=\frac{y_{1}(T)}{\sqrt{\sigma^{2}-4}} \cdot \frac{\sigma}{\cos \theta_{1}(T-)} \geq \frac{\sigma^{2}}{\sqrt{\left(\sigma^{2}-4\right)\left(\sigma^{2}-16\right)}}\left(s_{2}(T)-s_{1}(T)\right) .
$$

Comparing (6.17) with (6.16), it is clear that the value of time has a downward jump at the time $t=T$ when the $\operatorname{arcs} \gamma_{3}$ and $\gamma_{4}$ meet.

Example 6.2. We again consider the problem of minimizing the total area burned by the fire, assuming that walls constructed at a constant speed $\sigma>2$. Here we assume that at the initial time $t=0$ the fire occupies the unit disc $R_{0}=B_{1}$. Moreover, we assume that $F(x)=B_{1}$ for all $x \in \mathbb{R}^{2}$, so that the fire propagates at unit speed in all directions.

As described in [4], an optimal strategy is as follows. First construct an arc of circumference $\Gamma_{1}$. Then construct two arcs of logarithmic spirals $\Gamma_{2}, \Gamma_{3}$ along the boundary of the burned region (see Fig. 11). In this case, $\Gamma_{1}$ is a free arc, while $\Gamma_{2}, \Gamma_{3}$ are boundary arcs. Here the length of the $\operatorname{arc} \Gamma_{1}$ should satisfy

$$
m_{1}\left(\Gamma_{1}\right)=\sigma \cdot d\left(Q_{2}, R_{0}\right)=\sigma \cdot d\left(Q_{3}, R_{0}\right),
$$




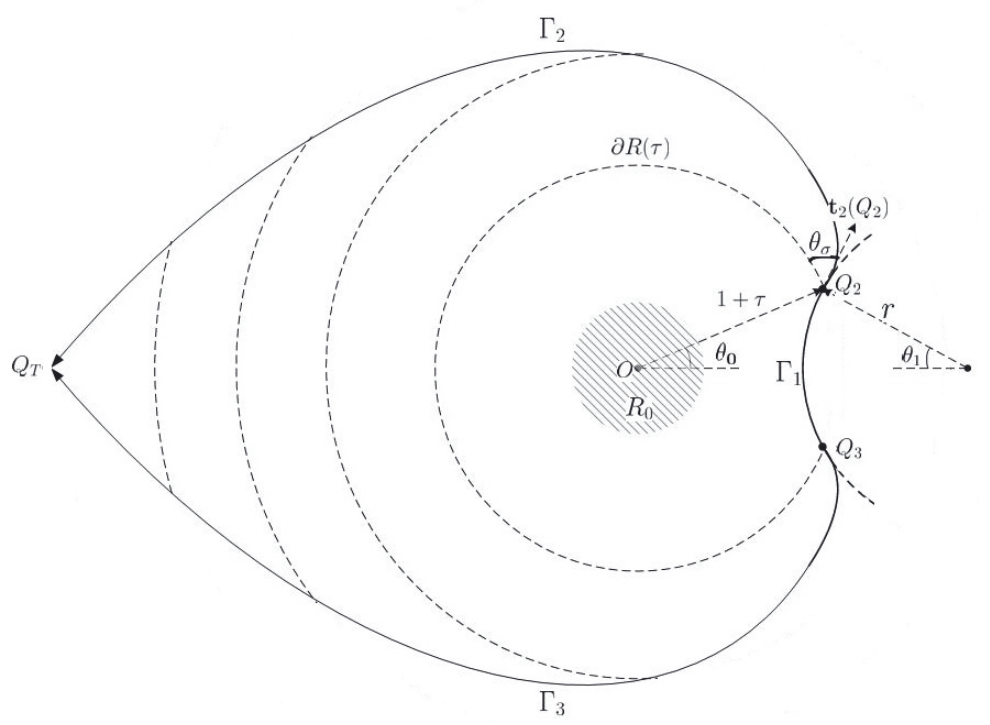

FIgURE 11. The burned region is enclosed by the arc of circumference $\Gamma_{1}$ and by two arcs of logarithmic spirals $\Gamma_{2}, \Gamma_{3}$.

so that the two end-points $Q_{2}, Q_{3}$ are reached by the boundary of the burned region $R^{\gamma}(\tau)$ exactly at the time $\tau$ when the construction of the arc $\Gamma_{1}$ is completed. According to Theorem 5.1, the junctions at $Q_{2}$ and at $Q_{3}$ must be $\mathcal{C}^{1}$, i.e. the arcs must join tangentially. For each time $\tau$, the above conditions determine a unique strategy $\Gamma^{(\tau)}$. These conditions reduce the problem to an optimization problem over the scalar parameter $\tau$.

We parameterize the boundary arcs $\Gamma_{2}, \Gamma_{3}$ by the time $t$, using polar coordinates. The radius is $\rho(t)=1+t$, while the angle $\theta(t)$ is the angle between $O \Gamma_{i}$ and the axis of symmetry. As shown in Figure 11, $r$ denotes the radius of the arc of circumstance $\Gamma_{1}$, while $\theta_{1}$ denotes half of the corresponding angle. Moreover, $\theta_{0}=\theta(\tau)$ is the angular coordinate of the point of junction $Q_{2}$, while

$$
\theta_{\sigma}=\arcsin \left(\frac{2}{\sigma}\right)
$$

denotes the constant angle between the arcs of spirals $\Gamma_{2}, \Gamma_{3}$ and the circumferences centered at the origin. At the junction point $Q_{2}$ we have the identities

$$
\left\{\begin{aligned}
\theta_{\sigma} & =\theta_{0}+\theta_{1} \\
\sigma \cdot \tau & =2 r \theta_{1} \\
r \cdot \sin \theta_{1} & =(1+\tau) \cdot \sin \theta_{0} .
\end{aligned}\right.
$$

In (6.18), $\theta_{0}$ and $r$ can be written in terms of $\theta_{1}$ from the first two equations. Inserting these values in the last equation, the system reduces to the single equation

$$
\Phi\left(\theta_{1}\right) \doteq \frac{\sigma \tau}{2 \theta_{1}} \cdot \sin \theta_{1}-(1+\tau) \cdot \sin \left(\theta_{\sigma}-\theta_{1}\right)=0
$$


We claim that under the assumption $\tau<\tau_{\sigma} \doteq \frac{4}{\sigma^{2}-4}$, the function $\Phi$ has unique root $\theta_{1} \in\left[0, \theta_{\sigma}\right]$. Indeed, the assumption implies $\frac{\sigma \tau}{2}<(1+\tau) \sin \theta_{\sigma}$, hence $\Phi(0)<0$. A differentiation yields

$$
\begin{aligned}
\frac{\mathrm{d}}{\mathrm{d} \theta_{1}} \Phi\left(\theta_{1}\right) & =\frac{\sigma \tau \cos \theta_{1}}{2 \theta_{1}^{2}} \cdot\left(\theta_{1}-\tan \theta_{1}\right)+(1+\tau) \cdot \cos \left(\theta_{\sigma}-\theta_{1}\right) \\
& =\frac{\sigma \tau \cos \theta_{1}}{2 \theta_{1}^{2}} \cdot\left(\theta_{1}-\tan \theta_{1}\right)+(1+\tau) \cdot\left(\cos \theta_{\sigma} \cos \theta_{1}+\sin \theta_{\sigma} \sin \theta_{1}\right) \\
& \geq \frac{\sigma \tau \cos \theta_{1}}{2 \theta_{1}^{2}} \cdot\left(\theta_{1}-\tan \theta_{1}\right)+(1+\tau) \sin \theta_{\sigma} \sin \theta_{1} \\
& \geq \frac{\sigma \tau \cos \theta_{1}}{2 \theta_{1}^{2}} \cdot\left(\theta_{1}-\tan \theta_{1}\right)+\frac{\sigma \tau}{2} \sin \theta_{1} \\
& \geq \frac{\sigma \tau \cos \theta_{1}}{2 \theta_{1}^{2}} \cdot\left(\sin \theta_{1}-\tan \theta_{1}\right)+\frac{\sigma \tau}{2} \sin \theta_{1} \\
& =\frac{\sigma \tau \sin \theta_{1}}{2 \theta_{1}^{2}} \cdot\left(\cos \theta_{1}-1+\theta_{1}^{2}\right)>0, \quad \text { for all } \theta_{1} \in(0, \pi / 2),
\end{aligned}
$$

showing that $\Phi$ strictly increases.

Moreover, $\Phi\left(\theta_{\sigma}\right)=\frac{\sigma \tau}{2 \theta_{\sigma}} \cdot \sin \theta_{\sigma}>0$, and $\Phi(0)<0$. Hence $\Phi$ has unique zero inside the interval $] 0, \theta_{\sigma}[$.

If $\tau$ is given and sufficiently small, the parameters $\theta_{0}, \theta_{1}$ and $r$ are thus uniquely determined by the system (6.18). From now on, we consider them as functions of $\tau$. The terminal time $T$ can be determined implicitly from the identity

$$
\pi-\theta_{0}=\int_{\tau}^{T} \frac{\sqrt{(\sigma / 2)^{2}-1}}{1+t} \mathrm{~d} t=\sqrt{(\sigma / 2)^{2}-1} \cdot \ln \left(\frac{1+T}{1+\tau}\right) .
$$

The total area burned by the fire is computed by

$$
A(\tau) \doteq \int_{\tau}^{T}(1+t)^{2} \cdot \frac{\sqrt{\sigma^{2} / 4-1}}{1+t} \mathrm{~d} t+(1+\tau)^{2} \cdot \sin \theta_{0} \cos \theta_{0}-r^{2} \cdot\left(\theta_{1}-\sin \theta_{1} \cos \theta_{1}\right) .
$$

Notice that this expression can be regarded as a function of the scalar variable $\tau$. In order to find the optimal strategy, one could simply minimize (6.21) w.r.t. the scalar variable $\tau$.

Alternatively, one can determine the optimal value of $\tau$ from a matching condition. Indeed, as proved in Theorem 5.1, the value of time must be a continuous function, constant for $t \in[0, \tau]$, then decreasing to zero for $t \in[\tau, T]$. Along the free $\operatorname{arc} \Gamma_{1}$, one has

$$
W(t)=\sigma r \quad t \in[0, \tau] .
$$

To compute the value of time along the boundary $\operatorname{arcs} \Gamma_{2}, \Gamma_{3}$, we need to determine by how much a small perturbation of the data can increase the total cost. Namely, assume that at time $t$ we shift the edge of the wall $\gamma_{2}(t)$ along the boundary of the burned region. Working in polar coordinates, this means that at time $t$, instead of being at $(\rho, \theta)=(1+t, \theta(t))$, we move it to the point

$$
(\rho, \theta)=\left(1+t, \theta_{\varepsilon}(t)\right) \doteq\left(1+t, \theta(t)-\frac{\varepsilon}{1+t}\right) .
$$

The rate of increase of the corresponding burned area is measured by

$$
\begin{aligned}
q_{2}(t) & =\lim _{\varepsilon \rightarrow 0} \frac{1}{\varepsilon} \int_{t}^{T} \rho(s) \cdot\left(\theta(s)-\theta_{\varepsilon}(s)\right) \mathrm{d} s \\
& =\int_{t}^{T} \frac{1+s}{1+t} \mathrm{~d} s=\frac{(1+T)^{2}-(1+t)^{2}}{2(1+t)} .
\end{aligned}
$$


By (4.30), the value of time along the boundary $\operatorname{arc} \Gamma_{2}$ is

$$
W(t)=\frac{\sigma q_{2}(t)}{\cos \theta_{\sigma}}=\frac{\sigma^{2}}{\sqrt{\sigma^{2}-4}} \cdot\left\{\frac{(1+T)^{2}}{2(1+t)}-\frac{1+t}{2}\right\} \quad t \in[\tau, T]
$$

Of course, the same value is valid along the boundary $\operatorname{arc} \Gamma_{3}$. It is easily checked that the above function is monotonically decreasing and vanishes for $t=T$.

Imposing that the value of time along the free arc and the boundary arcs coincide at the junction time, i.e. that the right hand sides in (6.22)-(6.23) coincide at time $t=\tau$, we obtain an additional equation to determine $\tau$. The following table displays the various parameters of an optimal strategy, which have been numerically computed for different values of the construction speed $\sigma$. Clearly, as the construction speed increases, the time $T$ needed to block the fire and the total burned area $A$ decrease.

\begin{tabular}{|c|c|c|c|c|c|c|c|}
\hline$\sigma$ & $\tau_{\sigma}$ & $\tau$ & $\theta_{0}$ & $r$ & $\theta_{1}$ & $T$ & $A(\tau)$ \\
\hline 2.2 & 4.76190 & 4.76037 & 1.14097 & 42772.4 & 0.00012 & 452.370 & 47126.4 \\
\hline 2.4 & 2.27273 & 2.26594 & 0.98373 & 1973.44 & 0.00138 & 83.4908 & 2374.59 \\
\hline 3.0 & 0.80000 & 0.77928 & 0.71676 & 90.1463 & 0.01297 & 14.5653 & 135.926 \\
\hline 4.0 & 0.33333 & 0.30351 & 0.48427 & 15.4335 & 0.03933 & 5.04522 & 30.9903 \\
\hline 6.0 & 0.12500 & 0.09588 & 0.26533 & 3.86080 & 0.07450 & 2.02972 & 11.5975 \\
\hline 8.0 & 0.06667 & 0.04295 & 0.16528 & 1.96597 & 0.08740 & 1.24912 & 7.86743 \\
\hline 10.0 & 0.04167 & 0.02286 & 0.11180 & 1.27599 & 0.08956 & 0.89847 & 6.38192 \\
\hline
\end{tabular}

Acknowledgements. This work was supported by NSF through grant DMS-0807420, "New problems in nonlinear control".

\section{REFERENCES}

[1] J.P. Aubin and A. Cellina, Differential inclusions. Springer-Verlag, Berlin (1984).

[2] M. Bardi and I. Capuzzo-Dolcetta, Optimal control and viscosity solutions of Hamilton-Jacobi-Bellman equations. Birkhäuser, Boston (1997).

[3] V.G. Boltyanskii, Sufficient conditions for optimality and the justification of the dynamic programming principle. SIAM J. Control Optim. 4 (1966) 326-361.

[4] A. Bressan, Differential inclusions and the control of forest fires. J. Differ. Equ. 243 (2007) 179-207.

[5] A. Bressan and C. De Lellis, Existence of optimal strategies for a fire confinement problem. Comm. Pure Appl. Math. 62 (2009) 789-830.

[6] A. Bressan and Y. Hong, Optimal control problems on stratified domains. NHM 2 (2007) 313-331.

[7] A. Bressan and B. Piccoli, Introduction to the Mathematical Theory of Control, AIMS Series in Applied Mathematics 2. AIMS, Springfield Mo. (2007).

[8] A. Bressan and T. Wang, The minimum speed for a blocking problem on the half plane. J. Math. Anal. Appl. 356 (2009) 133-144.

[9] A. Bressan and T. Wang, Equivalent formulation and numerical analysis of a fire confinement problem. ESAIM: COCV 16 (2010) 974-1001.

[10] A. Bressan, M. Burago, A. Friend and J. Jou, Blocking strategies for a fire control problem. Analysis and Applications 6 (2008) 229-246.

[11] P. Brunovský, Every normal linear system has a regular time-optimal synthesis. Math. Slovaca 28 (1978) 81-100.

[12] F.H. Clarke, Optimization and Nonsmooth Analysis. Second edition, SIAM, Philadelphia (1990).

[13] W.H. Fleming and R.W. Rishel, Deterministic and Stochastic Optimal Control. Springer-Verlag, New York (1975).

[14] A.D. Ioffe and V.M. Tihomirov, Theory of Extremal Problems. North-Holland, New York (1974) 233-235.

[15] R. Vinter, Optimal Control. Birkhäuser, Boston (2000). 